\title{
Association of Structural Magnetic Resonance Imaging Measures With Psychosis Onset in Individuals at Clinical High Risk for Developing Psychosis
}

Citation for published version (APA):

Jalbrzikowski, M., Hayes, R. A., Wood, S. J., Nordholm, D., Zhou, J. H., Fusar-Poli, P., Uhlhaas, P. J., Takahashi, T., Sugranyes, G., Kwak, Y. B., Mathalon, D. H., Katagiri, N., Hooker, C. I., Smigielski, L., Colibazzi, T., Via, E., Tang, J. S., Koike, S., Rasser, P. E., ... van Amelsvoort, T. (2021). Association of Structural Magnetic Resonance Imaging Measures With Psychosis Onset in Individuals at Clinical High Risk for Developing Psychosis: An ENIGMA Working Group Mega-analysis. JAMA Psychiatry, 78(7), 753766. https://doi.org/10.1001/jamapsychiatry.2021.0638

Document status and date:

Published: 01/07/2021

DOI:

10.1001/jamapsychiatry.2021.0638

Document Version:

Publisher's PDF, also known as Version of record

Document license:

Taverne

Please check the document version of this publication:

- A submitted manuscript is the version of the article upon submission and before peer-review. There can be important differences between the submitted version and the official published version of record. People interested in the research are advised to contact the author for the final version of the publication, or visit the DOI to the publisher's website.

- The final author version and the galley proof are versions of the publication after peer review.

- The final published version features the final layout of the paper including the volume, issue and page numbers.

Link to publication

\footnotetext{
General rights rights.

- You may freely distribute the URL identifying the publication in the public portal. please follow below link for the End User Agreement:

www.umlib.nl/taverne-license

Take down policy

If you believe that this document breaches copyright please contact us at:

repository@maastrichtuniversity.nl

providing details and we will investigate your claim.
}

Copyright and moral rights for the publications made accessible in the public portal are retained by the authors and/or other copyright owners and it is a condition of accessing publications that users recognise and abide by the legal requirements associated with these

- Users may download and print one copy of any publication from the public portal for the purpose of private study or research.

- You may not further distribute the material or use it for any profit-making activity or commercial gain

If the publication is distributed under the terms of Article 25fa of the Dutch Copyright Act, indicated by the "Taverne" license above, 


\section{Association of Structural Magnetic Resonance Imaging Measures With Psychosis Onset in Individuals at Clinical High Risk for Developing Psychosis An ENIGMA Working Group Mega-analysis}

ENIGMA Clinical High Risk for Psychosis Working Group

IMPORTANCE The ENIGMA clinical high risk (CHR) for psychosis initiative, the largest pooled neuroimaging sample of individuals at $\mathrm{CHR}$ to date, aims to discover robust neurobiological markers of psychosis risk.

OBJECTIVE To investigate baseline structural neuroimaging differences between individuals at $\mathrm{CHR}$ and healthy controls as well as between participants at CHR who later developed a psychotic disorder (CHR-PS+) and those who did not (CHR-PS-).

DESIGN, SETTING, AND PARTICIPANTS In this case-control study, baseline T1-weighted magnetic resonance imaging (MRI) data were pooled from 31 international sites participating in the ENIGMA Clinical High Risk for Psychosis Working Group. CHR status was assessed using the Comprehensive Assessment of At-Risk Mental States or Structured Interview for Prodromal Syndromes. MRI scans were processed using harmonized protocols and analyzed within a mega-analysis and meta-analysis framework from January to October 2020.

MAIN OUTCOMES AND MEASURES Measures of regional cortical thickness (CT), surface area, and subcortical volumes were extracted from T1-weighted MRI scans. Independent variables were group (CHR group vs control group) and conversion status (CHR-PS+ group vs CHR-PS- group vs control group).

RESULTS Of the 3169 included participants, 1428 (45.1\%) were female, and the mean (SD; range) age was 21.1 (4.9; 9.5-39.9) years. This study included 1792 individuals at CHR and 1377 healthy controls. Using longitudinal clinical information, 253 in the CHR-PS+ group, 1234 in the CHR-PS- group, and 305 at CHR without follow-up data were identified. Compared with healthy controls, individuals at CHR exhibited widespread lower CT measures (mean [range] Cohen $d=-0.13$ [-0.17 to -0.09$]$ ), but not surface area or subcortical volume. Lower CT measures in the fusiform, superior temporal, and paracentral regions were associated with psychosis conversion (mean Cohen $d=-0.22 ; 95 \% \mathrm{Cl},-0.35$ to 0.10 ). Among healthy controls, compared with those in the CHR-PS+ group, age showed a stronger negative association with left fusiform CT measures $(F=9.8 ; P<.001 ; q<.001)$ and left paracentral CT measures $(F=5.9 ; P=.005 ; q=.02)$. Effect sizes representing lower $C T$ associated with psychosis conversion resembled patterns of CT differences observed in ENIGMA studies of schizophrenia ( $\rho=0.35 ; 95 \% \mathrm{Cl}, 0.12$ to $0.55 ; P=.004)$ and individuals with 22q11.2 microdeletion syndrome and a psychotic disorder diagnosis $(\rho=0.43$; $95 \% \mathrm{Cl}, 0.20$ to $0.61 ; P=.001)$.

CONCLUSIONS AND RELEVANCE This study provides evidence for widespread subtle, lower CT measures in individuals at CHR. The pattern of CT measure differences in those in the CHR-PS+ group was similar to those reported in other large-scale investigations of psychosis. Additionally, a subset of these regions displayed abnormal age associations. Widespread disruptions in CT coupled with abnormal age associations in those at CHR may point to disruptions in postnatal brain developmental processes.

JAMA Psychiatry. 2021;78(7):753-766. doi:10.1001/jamapsychiatry.2021.0638 Published online May 5, 2021.
Supplemental content

Group Information: Members of the ENIGMA Clinical High Risk for Psychosis Working Group are listed at the end of this article.

Corresponding Authors: Maria Jalbrzikowski, PhD, University of Pittsburgh, 3501 Forbes Ave, Ste 430, Pittsburgh, PA 15213 (jalbrzikowskime @upmc.edu); Dennis Hernaus, PhD, Maastricht University, PO Box 616, 6200 MD, Maastricht, the Netherlands (dennis.hernaus@ maastrichtuniversity.nl). 
$\mathrm{T}$ he clinical high-risk (CHR) paradigm is a widely used framework to investigate mechanisms underlying psychosis vulnerability. Help-seeking individuals who do not meet diagnostic criteria for a psychotic disorder but typically present with subthreshold psychotic symptoms and accumulating risk factors are considered at CHR for developing psychosis. ${ }^{1}$ An estimated $18 \%$ to $20 \%$ of individuals at CHR develop a psychotic disorder within 2 years of identification, ${ }^{2}$ although conversion rates vary, likely due to heterogeneous recruitment and sampling strategies as well as interventions applied. ${ }^{3}$ However, despite decades of research, the nature of morphometrical differences associated with psychosis conversion remains largely unknown. Here, we aim to address this question by combining all available structural neuroimaging data in CHR to date in an attempt to better understand group differences associated with psychosis risk and conversion in this population.

A large body of work has used structural magnetic resonance imaging (sMRI) to investigate morphometric brain differences in individuals at CHR. ${ }^{4-20}$ However, the extent to which characteristic baseline (ie, when participants are initially ascertained and assessed at a first study visit) structural neuroimaging differences exist between those at CHR who later develop a psychotic disorder (CHR-PS+) compared with those who do not (CHR-PS-) is debated. Many studies failed to find baseline differences between these 2 groups, ${ }^{4,14,21,22}$ although a meta-analysis ${ }^{16}$ and multicenter study ${ }^{23}$ found lower prefrontal and temporal volumes or cortical thickness measured by MRI (which we will refer to as $C T$ ) in individuals at CHR who developed a psychotic disorder. High attrition rates in samples of individuals at $\mathrm{CHR}^{24}$ coupled with low psychosis conversion rates ${ }^{2,25}$ often yielded insufficient power to detect between-group structural brain differences. Moreover, small sample sizes can be associated with inflated effect sizes, ${ }^{26}$ so effect sizes of prior studies that found structural brain differences in individuals at CHR may be overestimated. Although multisite consortia aim to address these challenges, to our knowledge, the largest published sMRI studies to date included fewer than 50 individuals at CHR who later developed a psychotic disorder. ${ }^{21,23}$ Furthermore, it is currently unknown whether group differences are robust enough to predict outcomes.

Importantly, many participants at CHR are adolescents or young adults, a time frame associated with psychosis onset. ${ }^{27,28}$ Prefrontal-temporal brain regions, which are typically implicated in psychosis, show protracted developmental courses continuing through adolescence, ${ }^{29,30}$ suggesting that morphometric differences associated with psychosis risk vary with age. Indeed, there are developmental influences on psychotic symptom presentation, ${ }^{31}$ perhaps driven by differences in regional brain changes. It is not fully understood how age-related patterns in brain morphometry in individuals at CHR differ from normal development. Thus, using a developmental framework to examine whether morphometric differences in individuals at CHR are influenced by age may provide important insights into mechanisms associated with psychosis risk and the stability of neuroimaging measures associated with psychosis risk across development.

\section{Key Points}

Question How are brain morphometric features associated with later psychosis conversion in individuals at clinical high risk (CHR) for developing psychosis?

Findings In this case-control study including 3169 participants, lower cortical thickness, but not cortical surface area or subcortical volume, was more pronounced in individuals at CHR in a manner highly consistent with thinner cortex in individuals with established psychosis. Regions that displayed lower cortical thickness in individuals at CHR who later developed a psychotic disorder additionally displayed abnormal associations with age.

Meaning In this study, CHR status and later transition to psychosis was robustly associated with lower cortical thickness; abnormal age associations and specificity to cortical thickness may point to aberrant postnatal brain development in individuals at CHR, including pruning and myelination.

Finally, to our knowledge, it is unknown whether baseline brain differences associated with future conversion to psychosis resemble those observed in other large-scale psychosis studies. Understanding whether morphometric differences in individuals at CHR overlap with those observed in individuals who have schizophrenia ${ }^{32,33}$ and individuals with a genetic subtype of psychosis ${ }^{34,35}$ will provide insights into convergent or distinct differences across the psychosis spectrum.

To address these questions, we founded the Enhancing Neuro Imaging Genetics Through Meta-Analysis (ENIGMA) Clinical High Risk for Psychosis Working Group in 2018. Using baseline sMRI data and longitudinal clinical information from 31 sites, this study addressed the following questions:

1. Do participants at CHR and healthy controls differ in CT, surface area (SA), and/or subcortical volumes?

2. Is there a neuroanatomic signature associated with future transition to a psychotic disorder (CHR-PS+ group vs CHR-PS- group vs control group)?

3. Do structural neuroimaging measures identified in aims 1 and 2 display group differences in age associations suggestive of abnormal developmental trajectories?

4. Is the pattern of morphometric alteration associated with psychosis conversion similar to that observed in other ENIGMA studies of psychosis?

\section{Methods}

\section{Participants}

We included 1792 individuals at CHR, including 253 in the CHR-PS+ group, 1234 in the CHR-PS- group, and 305 without follow-up data, and 1377 healthy controls from 31 sites participating in the ENIGMA Clinical High Risk for Psychosis Working Group (Table). Participants met the Comprehensive Assessment of At-Risk Mental States (CAARMS; $n=821$ ) or Structured Interview for Prodromal Syndromes (SIPS; $\mathrm{n}=971$ ) CHR criteria (eMethods in the Supplement). Site-specific inclusion and exclusion criteria are detailed in eTable 1 in the Supplement. All sites obtained local institutional review board approval prior to data collection. Informed written consent was 


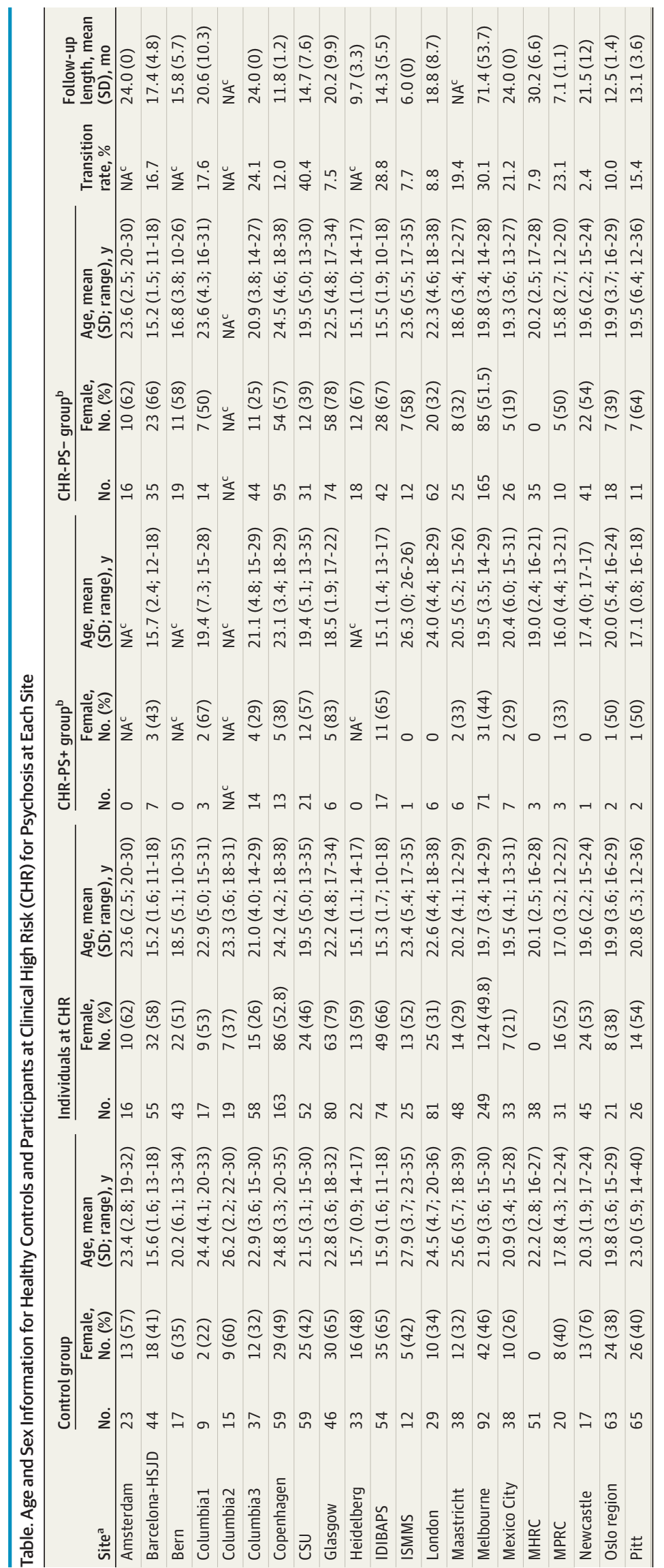

obtained from every participant or the participant's guardian for participants younger than 18 years. All studies were conducted in accordance with the Declaration of Helsinki. ${ }^{36}$

\section{Image Acquisition and Processing}

A total of 31 sites contributed T1-weighted MRI brain scans from 50 MRI scanners, including 42 3-T scanners and 81.5-T scanners (eTable 2 in the Supplement). Scanners were manufactured by Siemens $(n=23)$, Philips $(n=8)$, GE ( $n=18)$, and Toshiba $(n=1)$. A breakdown of the number of scans obtained for those in the CHR-PS+, CHR-PS-, and control groups for each scanner is reported in eTable 3 in the Supplement. After processing the data using Freesurfer analysis software (eTable 2 in the Supplement), ${ }^{37-39}$ we extracted 68 CT, 68 SA, and 16 subcortical volume measures. We also examined 3 global neuroimaging measurestotal intracranial volume (ICV), mean CT, and total SA-resulting in 155 neuroimaging measures. We implemented the ENIGMA consortium quality assessment pipeline. ${ }^{32-35,40,41}$ A priori power calculations are included in eMethods in the Supplement.

\section{Statistical Analyses \\ Group-Related and Conversion-Related Differences in sMRI Metrics}

We assessed group differences using general linear models (GLMs) within a mega-analysis framework, with each sMRI measure (ie, CT, SA, or subcortical volume) as the dependent variable and group (CHR or healthy control) or conversion status (CHR-PS+, CHR-PS-, or control) as the independent variable. We included age, age ${ }^{2}$, sex, and estimated total ICV as covariates in all models and corrected for multiple comparisons ( $n=155$ ) using the false discovery rate method. ${ }^{42} q$ Values less than .05 were considered statistically significant. Significance for $P$ values for minimaleffects testing was set at $P<.05$ and were 1-tailed; all other $P$ and $q$ values were 2-tailed. All analyses were conducted using $\mathrm{R}$ version 3.6.3 (The R Foundation).

For all structural neuroimaging measures, we calculated Cohen $d$ effect sizes from the GLMs between 2 (individuals at CHR vs healthy controls) or 3 groups of interest (CHR-PS+ group vs control group; CHR-PS+ group vs CHR-PSgroup; CHR-PS- group vs control group). Based on recent work demonstrating that neuroComBat harmonization increases statistical power within a mega-analytic framework, ${ }^{43}$ primary analyses were conducted within a megaanalysis framework using data that were cor- 


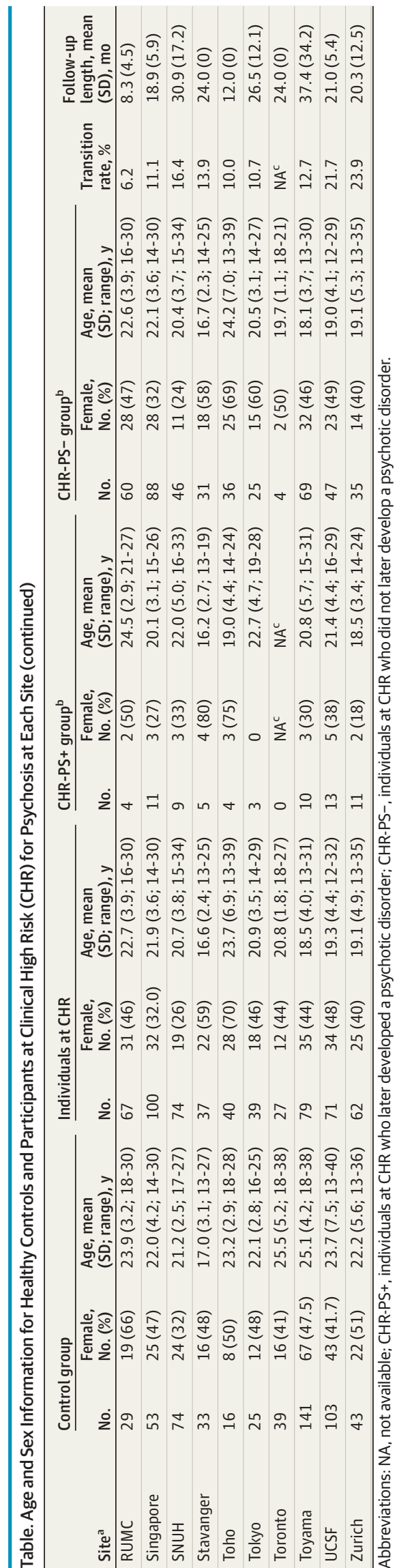

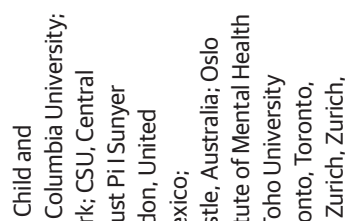

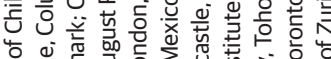

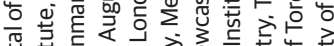

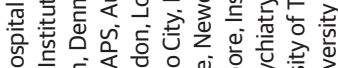

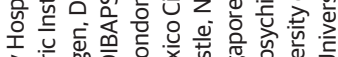

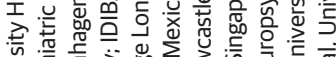

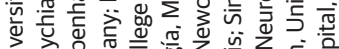

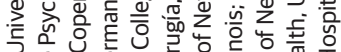

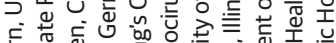

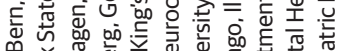

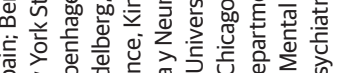
足

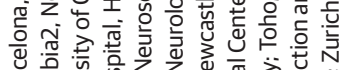
D.
D.

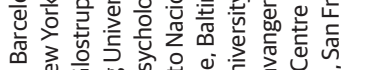

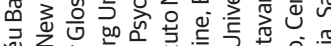

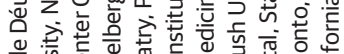

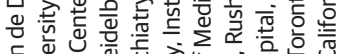

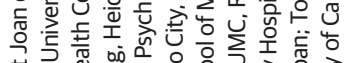

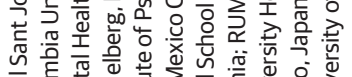

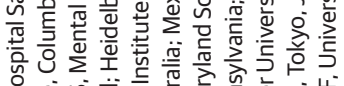

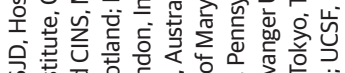

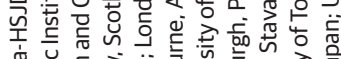

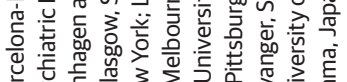

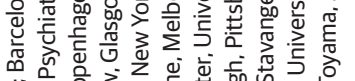

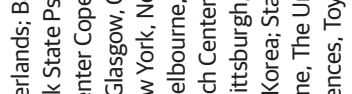

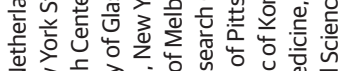

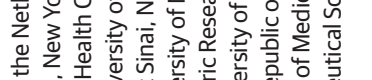

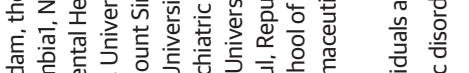

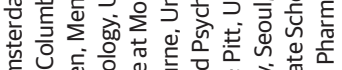

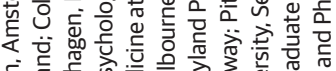

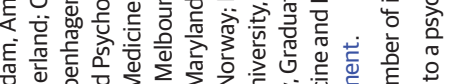

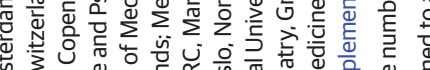

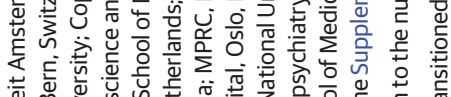

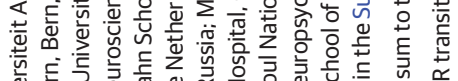

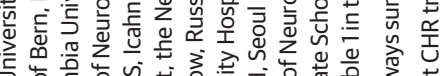

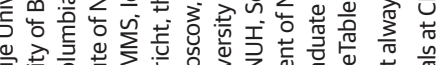

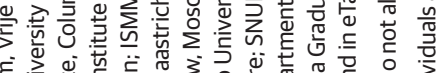

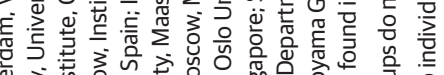

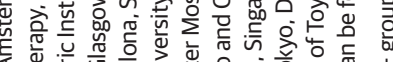

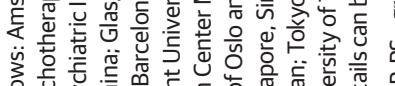

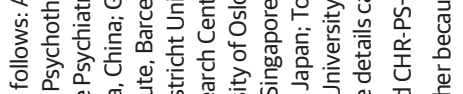

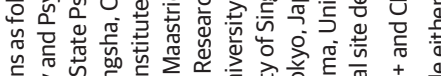

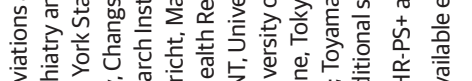

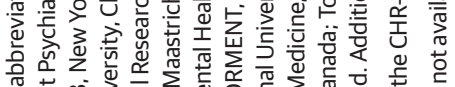

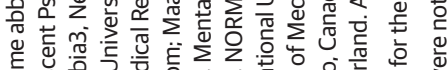

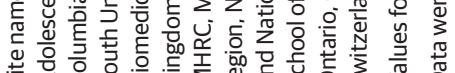

rected for site and scanner associations using neuroComBat harmonization. Additional analyses were conducted to assess the robustness of results obtained using this approach (eMethods in the Supplement). For all neuroimaging measures, we investigated sMRI differences associated with the specific psychosisrisk syndromes (eg, attenuated positive symptom syndrome) (eMethods in the Supplement).

To evaluate the stability of group and conversion status differences, we performed analyses statistically controlling for baseline psychotropic medication exposure. To assess site effects, we conducted jackknife resampling analyses, ie, iteratively removing one site's data and rerunning respective analyses. ${ }^{44}$ sMRI measures that failed to show a group or conversion status association at a $q$ value less than .05 in more than $10 \%$ jackknife iterations (ie, 4 of 31 sites) were considered unstable.

To assess the meaningfulness of obtained effect sizes, we used 2 analytic approaches: equivalence testing (to assess whether observed differences fell within the upper and lower bounds of a predefined smallest effect size of interest, providing support for the absence of a meaningful effect) and minimal-effects testing (to assess whether observed effects were greater than the same predefined effect size). ${ }^{45}$ Upper and lower bounds (representing the positive and negative predefined smallest effect size of interest) were set to a Cohen $d$ of 0.15 and -0.15 , respectively (eMethods in the Supplement).

\section{Group and Conversion-Related Differences in sMRI Age Associations}

We used general additive models (GAMs) ${ }^{46,47}$ to model group and conversion status differences in the association between age and sMRI measures (eMethods in the Supplement). First, we examined the interaction between group (individuals at CHR vs healthy controls) and age in the 56 neuroimaging measures that differed at a $q$ value less than .05 between healthy controls and individuals at CHR. Next, we conducted GAM analyses on the 4 sMRI measures on which the CHR-PS+, CHR-PS-, and control groups differed from each other (ie, left paracentral CT, right paracentral CT, left fusiform $\mathrm{CT}$, right superior temporal CT) in analy- 
ses of psychosis conversion. We examined the associations of baseline age and group/conversion status as well as the interaction between the 2 variables. Sex and estimated ICV were included as covariates. Similar to previous work examining age associations during adolescent development, ${ }^{48,49}$ we restricted our sample's age range to 12 to 25 years (eTable 4 in the Supplement). Details on post hoc analyses for significant interaction associations are provided in the eMethods in the Supplement.

\section{Comparison of Psychosis Conversion Effect Sizes With Findings of Other ENIGMA Studies}

We computed Spearman rank correlations to assess the extent to which the pattern of observed effect sizes (Cohen $d$ for CHR-PS+ and CHR-PS- groups vs control group) correlated with the pattern found in prior psychosis studies, specifically the ENIGMA Schizophrenia Working Group (individuals with schizophrenia vs healthy controls) ${ }^{32,33}$ and ENIGMA 22q11.2 Deletion Syndrome Working Group (individuals with 22q11.2 deletion syndrome with psychosis vs those with 22q11.2 deletion syndrome without psychosis). ${ }^{34,35}$ As a control, we compared the effect sizes of the CHR-PS+ group and CHR-PS- group vs control group with the effect sizes of the major depressive disorder (MDD) group vs control group published by the ENIGMA Major Depressive Disorder Working Group (eMethods in the Supplement). ${ }^{40,41}$

\section{Results}

Sample Characteristics

Of the 3169 included participants, 1428 (45.1\%) were female, and the mean (SD; range) age was 21.1 (4.9; 9.5-39.9) years (Table). Intelligence quotient (IQ) comparisons between healthy controls and individuals at CHR are reported in eTable 5 in the Supplement. Within each site, baseline IQ measures were largely similar in all participants at CHR, including those in the CHR-PS+ group, CHR-PS- group, and participants at CHR without follow-up information (eTable 6 in the Supplement). For symptom measures, participants at CHR without follow-up data had less severe baseline positive, negative, and disorganized symptoms on the SIPS compared with participants in the CHR-PS+ and CHR-PS- groups (eTable 7 in the Supplement). Compared with the CHR-PS+ and CHR-PS- groups, those without follow-up data had less severe cognitive changes on the CAARMS (eTable 7 in the Supplement). Few participants at CHR reported typical (less than 1\%) and/or atypical (12.4\%) antipsychotic medication use (eTable 8 in the Supplement).

\section{CT in Participants at CHR vs Healthy Controls}

In neuroComBat-harmonized GLM mega-analyses, participants at CHR had smaller global neuroimaging measures compared with healthy controls (estimated ICV: Cohen $d=-0.13$; 95\% CI, -0.20 to -0.06 ; mean CT: Cohen $d=-0.18$; 95\% CI, -0.25 to -0.11 ; total SA: Cohen $d=-0.15$; $95 \% \mathrm{CI},-0.22$ to -0.08). We also observed significant group associations in 53 additional GLMs (eTable 9 in the Supplement). The largest group associations were observed for widespread lower CT in individuals at CHR vs healthy controls (42 of 68 comparisons; Cohen $d$ range, -0.17 to -0.09) (Figure 1A; eTable 9 and eFigure 1 in the Supplement). Few subcortical (3 of 16) and SA (8 of 68) group differences were observed. No group $\times$ sex interactions were detected.

We present results of possible confound analyses, including ICV, medication and site associations, equivalence testing, and results of neuroComBat harmonization in eTables 10 to 13 and eFigures 1 and 2 in the Supplement. No sMRI measures were uniquely sensitive to psychosis-risk syndrome (eResults and eTables 14 to 17 in the Supplement).

\section{Association of Paracentral, Fusiform, and Superior Temporal} CT With Psychosis Conversion

A total of 48 structural neuroimaging measures exhibited a significant overall association with psychosis conversion status in GLM mega-analyses using neuroComBat harmonized data (Figure 1B; eTable 18 and eFigure 3 in the Supplement). Most significant differences were observed for CT measures $(n=37)$. Within these 48 regions, we conducted pairwise GLMs between the CHR-PS+ and control groups, CHR-PS- and control groups, and CHR-PS+ and CHR-PS- groups. Of these 48 regions, the CHR-PS+ group differed from the CHR-PS- and control groups on 4 neuroimaging measures.

Compared with the control and CHR-PS- groups, the CHR-PS+ group exhibited lower CT in bilateral paracentral, right superior temporal, and left fusiform regions (mean Cohen $d$ of 4 sMRI measures $=-0.22 ; 95 \%$ CI, -0.35 to 0.10 ). Similar findings were observed for the left superior temporal and right fusiform regions. Moreover, the CHR-PS+ and CHRPS- groups exhibited thinner cortex in bilateral paracentral, superior temporal, and fusiform regions compared with the control group (Figure 2). Using minimal-effects testing, we observed that effect sizes for bilateral paracentral (left hemisphere: $z=-2.43 ; P=.02$; right hemisphere: $z=-1.86$; $P=.06)$, right superior temporal $(z=-2.29 ; P=.02)$, and left fusiform $(z=-2.00 ; P=.05)$ in the CHR-PS+ group vs the control group were all greater than 0.15 , except for the right paracentral region, underscoring the presence of notable group differences.

In all remaining comparisons of regions that exhibited a statistically significant association with psychosis conversion status, the CHR-PS+ and CHR-PS- groups significantly differed from the control group at $P<.05$. However, the CHR-PS+ group did not differ from the CHR-PS- group in any remaining comparisons (eTable18 in the Supplement). We observed no conversion status $\times$ sex interactions, and results remained stable when length of follow-up period was included as a covariate.

We present results of confound analyses (medication, site associations, equivalence testing) in the eResults, eTables 19 to 21, and eFigure 2 in the Supplement. There were no statistically significant psychosis risk syndrome $\times$ conversion status interactions (eResults and eTable 22 in the Supplement).

\section{Age Associations in CHR-PS+ and CHR-PS- Groups} Compared With Control Group

In GAM analyses, we observed no statistically significant group $\times$ age interactions for the 56 neuroimaging measures that differed 
A Effect sizes for healthy controls vs individuals at CHR

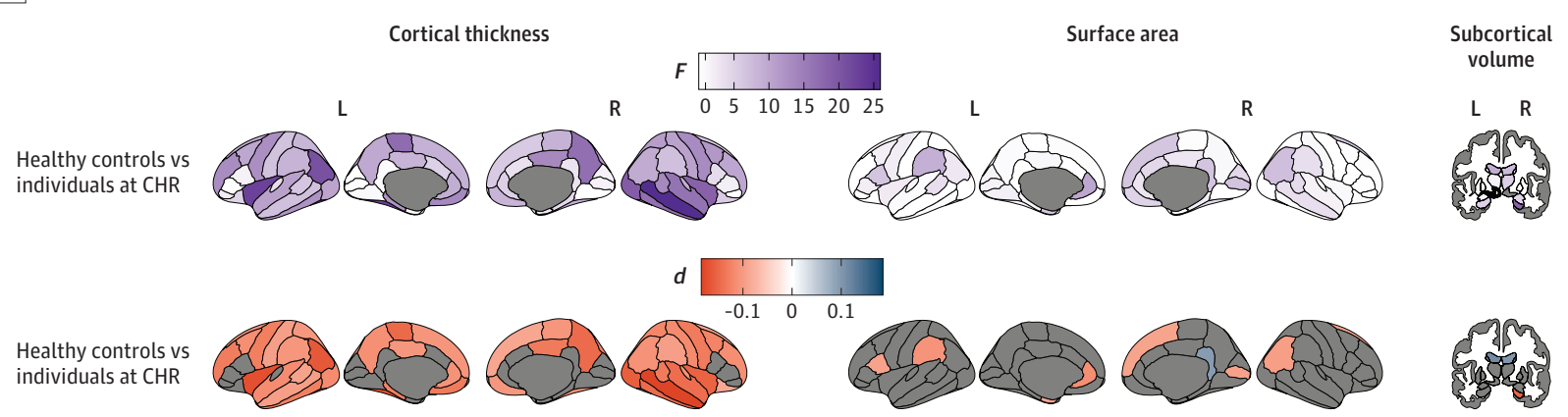

B Effect sizes for healthy controls vs CHR-PS+ vs CHR-PS-

Cortical thickness

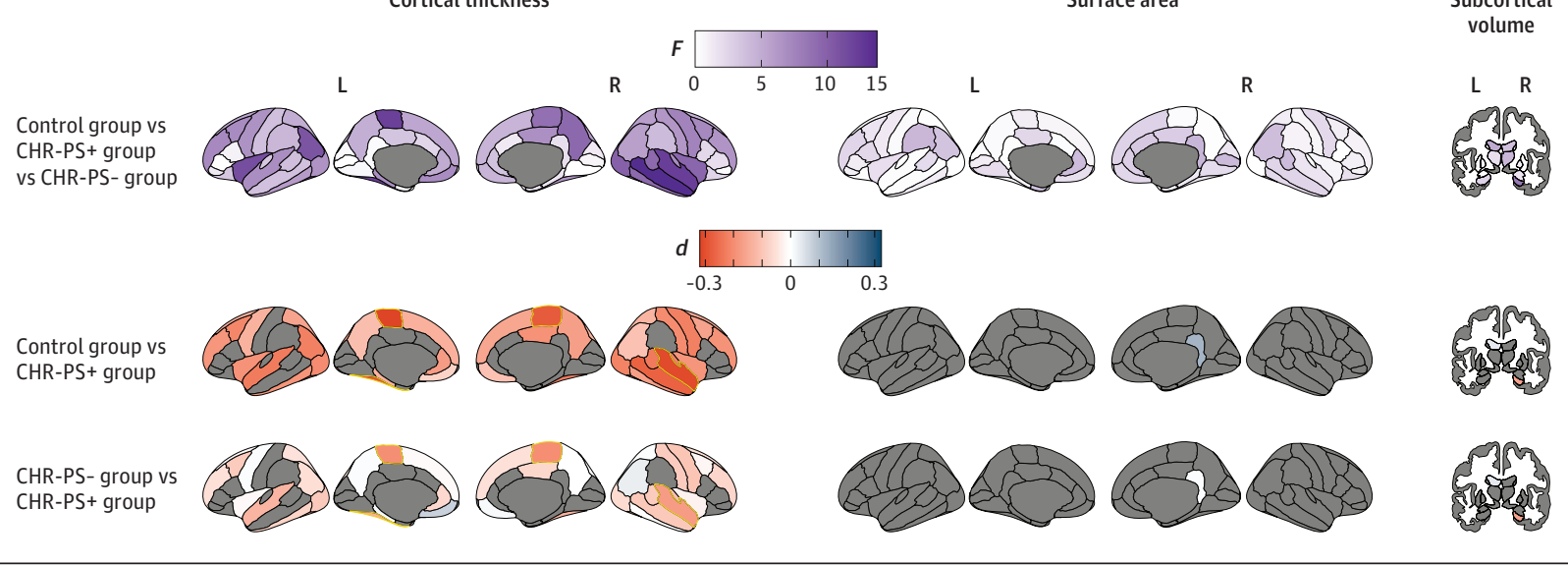

A, The top row reflects the results of the overall general linear model. A deeper purple indicates a greater group association (healthy controls vs individuals at clinical high risk [CHR]) in this region. We observed the greatest group associations in cortical thickness measures. The second row indicates the pairwise effect sizes for healthy controls vs individuals at CHR in regions that were statistically significant $(q<.05)$ in the overall comparison (top row). Regions that were not statistically significant in the overall comparison are gray. Compared with healthy controls, individuals at CHR exhibited lower cortical thickness across the cortex. Red indicates that healthy controls had a larger value compared with individuals at CHR for this region. B. The top row reflects the results of the overall general linear model. A deeper purple indicates a greater conversion status association (control group vs individuals at CHR who later developed a psychotic disorder [CHR-PS+] vs individuals at CHR who did not later develop a psychotic disorder [CHR-PS-]) in this region. The second and third rows indicate the pairwise effect sizes for the control group vs CHR-PS+ group and CHR-PS- group vs CHR-PS+ group, respectively. Pairwise comparisons are presented in regions that were statistically significant $(q<.05)$ in the overall comparison (top row). Regions that were not statistically significant in the overall comparison are gray. Regions in which the CHR-PS+ group had lower cortical thickness compared with the control group and the CHR-PS- group are highlighted in yellow.

between individuals at CHR and healthy controls (eTable 23 in the Supplement). We then conducted GAM analyses on the 4 SMRI measures on which the CHR-PS + group displayed lower CT compared with the CHR-PS- and control groups in psychosis conversion group analyses. These sMRI measures were left paracentral CT, right paracentral CT, left fusiform CT, and right superior temporal CT. Two measures displayed a significant psychosis conversion status $\times$ age interaction. For each group $\times$ age interaction analysis, we assessed group differences in age associations (ie, CHR-PS+ group vs control group, CHR-PS- group vs control group, and CHR-PS+ group vs CHR-PS- group).

In left fusiform, the association of age with CT differed between the CHR-PS+ group and control group ( $F=9.8$; $P<.001 ; q<.001)$ and the CHR-PS- group and control group ( $F=8.7 ; P<.001 ; q<.001$ ) (Figure $2 \mathrm{~A}$ ) but not between the CHR-PS+ group and CHR-PS- group $(F=1.3 ; P=.31 ; q=.45)$. Between ages 12 and 16 years, those in the control group showed a stronger negative association of age with CT compared with those in the CHR-PS+ and CHR-PS- groups. Although the interaction was not statistically significant, a similar pattern emerged for the right fusiform CT (Figure 3A; eTable 24 in the Supplement).

Age associations in the left paracentral CT differed between the CHR-PS+ group and the control group ( $F=5.9$; $P=.005 ; q=.02$ ) (Figure 2B) but not between the CHR-PSgroup and control group $(F=0.2 ; P=.68 ; q=.74)$ or the CHRPS+ group and CHR-PS- group $(F=1.9 ; P=.18 ; q=.45)$. Among individuals aged 12 to 15.8 years, those in the control group showed a stronger negative association of age with CT compared with those in the CHR-PS+ group. The association of age with CT did not differ between the CHR-PS- group and control group $(F=0.2 ; P=.69 ; q=.74)$. This pattern of results was not observed for the right paracentral CT (Figure 3B; eTable 24 in the Supplement). We found no significant age $\times$ conver- 
Figure 2. Bar Graphs for Regions in Which Individuals at Clinical High Risk Who Later Developed a Psychotic Disorder (CHR-PS+) Had Lower Cortical Thickness Compared With Those Who Did Not Later Develop a Psychotic Disorder (CHR-PS-) and Healthy Controls
A Fusiform cortical thickness
B Paracentral cortical thickness
C Superior temporal cortical thickness
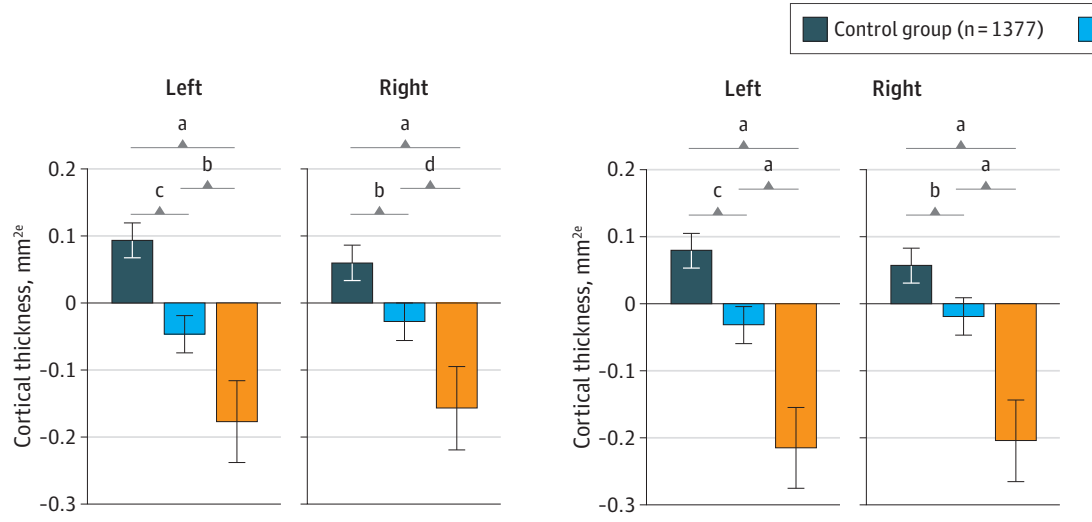

CHR-PS- group $(n=1234) \quad \square$ CHR-PS+ group $(n=253)$

${ }^{\mathrm{a}} P \leq .001$.

${ }^{\mathrm{d}} P<.10$.

${ }^{\mathrm{b}} P<.05$.

${ }^{\text {e }}$ Cortical thicknesses normalized to a mean of $\mathrm{O}$.

${ }^{\mathrm{c}} P \leq .01$.

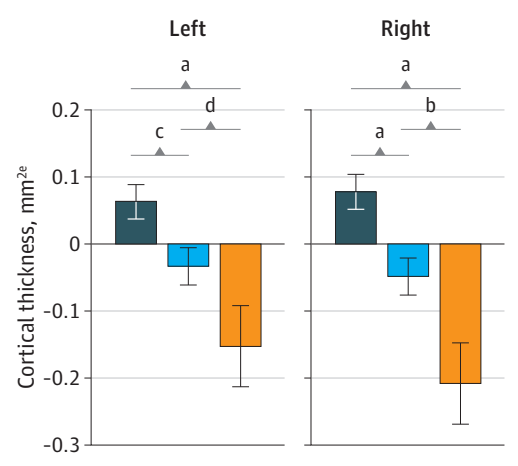

sion status interactions for the superior temporal CT (Figure 3C; eTable 24 in the Supplement); all groups showed negative associations of age with CT.

\section{CT in the ENIGMA CHR Group Compared With Other ENIGMA Working Groups}

eFigure 4 in the Supplement provides a visual overview of CT differences in the CHR-PS+ group, individuals with schizophrenia (using published data from the ENIGMA Schizophrenia Working Group ${ }^{32,33}$ ), and individuals with 22q11.2 deletion syndrome and a psychotic disorder (using published data from the ENIGMA 22q11.2 Deletion Syndrome Working Group $^{34,35}$ ).

The overall pattern of baseline CT differences in the CHR-PS+ group relative to the control group was significantly correlated with that observed in individuals with schizophrenia ( $\rho=0.35 ; 95 \%$ CI, 0.12 to $0.55 ; P=.004$ ) and in individuals with 22q11.2 deletion syndrome and psychosis $(\rho=0.43$; 95\% CI, 0.20 to 0.61; $P=.001$ ) (eFigure 4 in the Supplement). CT differences in the CHR-PS+ group relative to the control group were not correlated with CT differences observed in individuals with $\operatorname{MDD}(\rho=-0.03)$, and the slopes for the correlation between (1) CT differences in the CHR-PS+ group and individuals with schizophrenia and (2) CT differences in the CHR-PS+ group and individuals with MDD were significantly different (Steiger $z=2.06 ; P=.008$ ).

No significant correlations were observed for SA (schizophrenia: $\rho=-0.03$; 22q11.2 deletion syndrome with psychosis: $\rho=-0.06$ ) (eFigures 5 and 6 in the Supplement). Subcortical volume differences in the CHR-PS+ group relative to the control group were correlated with those observed in individuals with schizophrenia ( $\rho=0.54 ; P=.03$ ). A similar nonsignificant correlation was observed for the correlation analysis involving individuals with 22q11.2 deletion syndrome and psychosis $(\rho=0.46 ; P=.07$ ) (eFigures 5 and 6 in the
Supplement). Associations involving CHR-PS- vs control CT, SA, and subcortical volume differences were similar to those reported here (eResults in the Supplement).

\section{Discussion}

We conducted, to our knowledge, the largest multisite neuroimaging investigation to date in participants at CHR, examining baseline structural neuroimaging measures associated with later transition to psychosis. We found widespread lower CT in individuals at CHR, consistent with previously reported CT differences in individuals with an established psychotic disorder. Compared with those in the CHR-PS- and control groups, at baseline, those in the CHR-PS+ group exhibited thinner cortex in bilateral paracentral, right fusiform, and left superior temporal regions, with effect sizes significantly greater than what we considered to be meaningful a priori. Our results were robust to associations of medication exposure, sex, site, and length of follow-up period. Findings from this international effort suggest that conversion to psychosis among those at CHR is associated with lower CT at baseline.

We identified widespread regional lower CT in individuals at CHR compared with healthy controls. Lower CT has been observed in individuals with schizophrenia as well as other psychiatric disorders. ${ }^{32,40,50}$ Importantly, the overall pattern of lower CT in those in the CHR-PS+ and CHR-PS- groups resembled that observed in individuals with schizophrenia and individuals with 22q11.2 deletion syndrome and a psychotic disorder but not in individuals with MDD. For the CHR-PS+ group, correlations with CT differences in individuals with schizophrenia were significantly greater than the association observed with CT differences in individuals with MDD. Taken together, our results suggest that the overall constellation of reported CT differences in individuals at CHR resembles the 


\section{Figure 3. Age Associations of Regions That Exhibited an Association of Conversion Status}

A Age vs fusiform cortical thickness
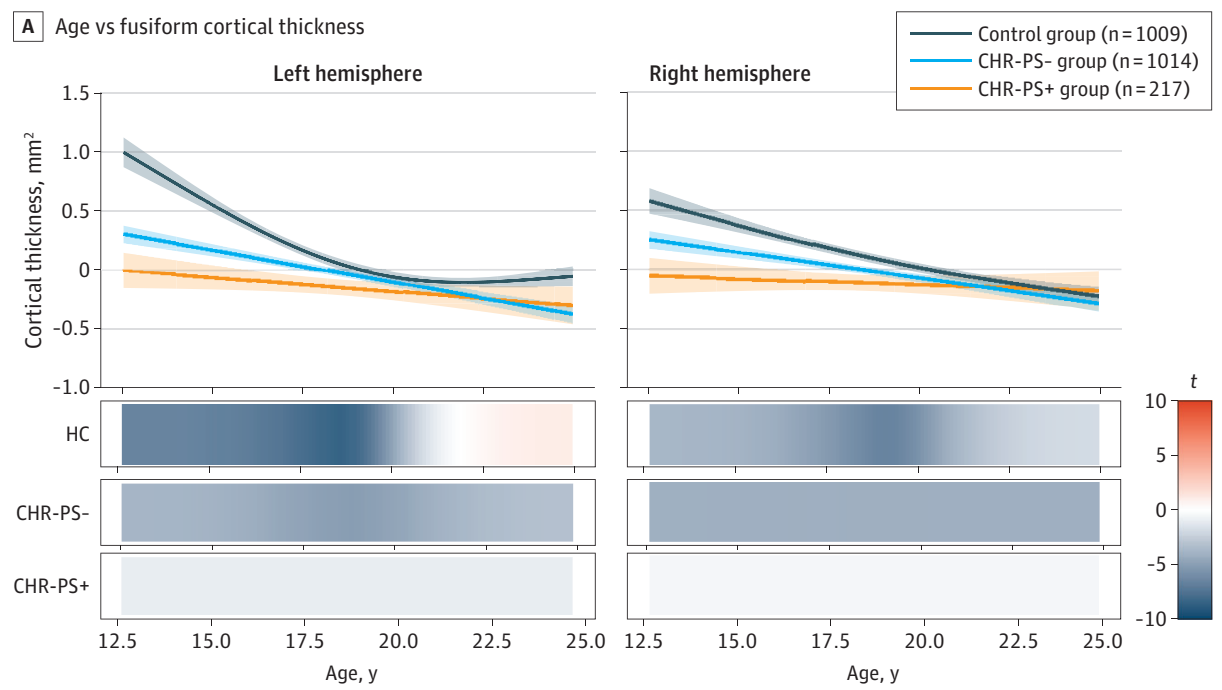

B Age vs paracentral thickness
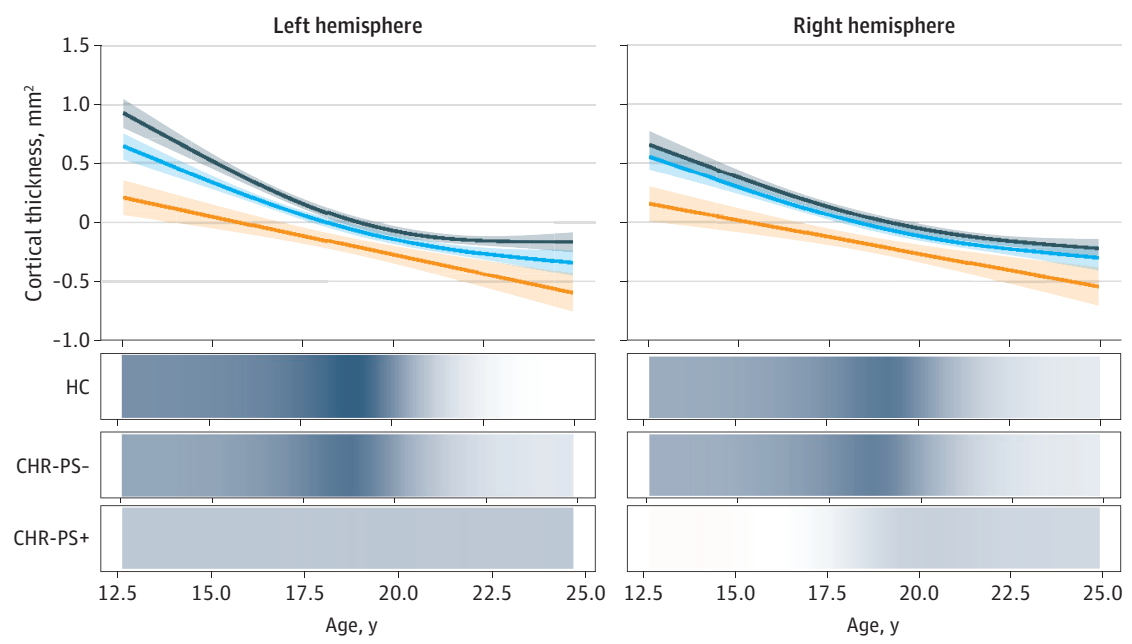

C Age vs superior temporal thickness
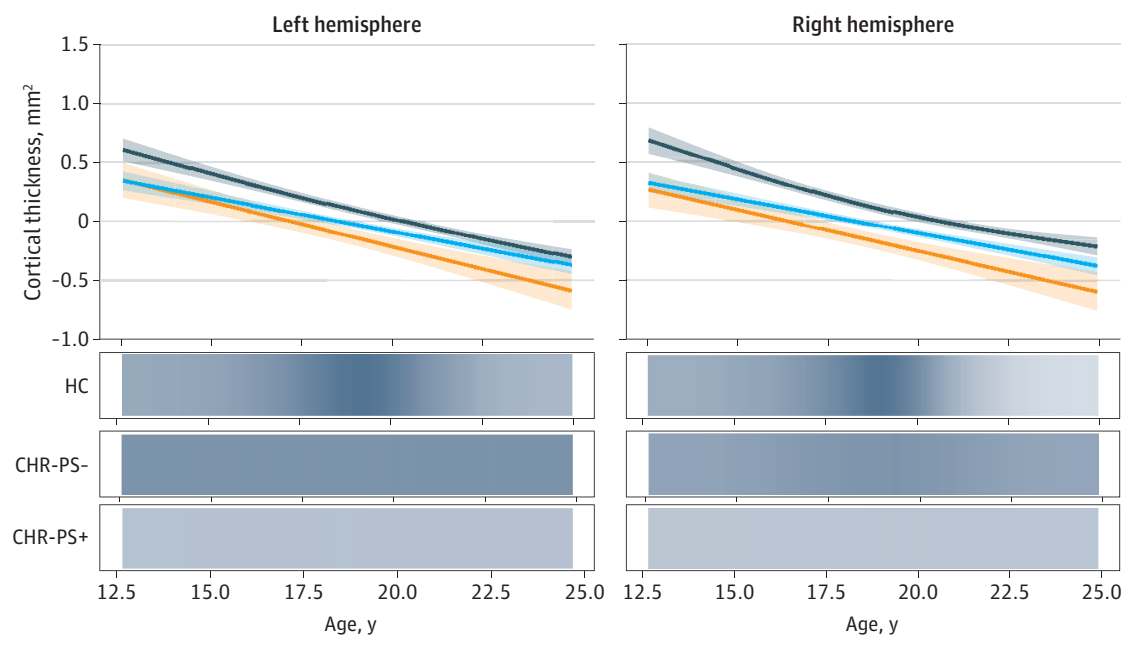

Each graph has a line of best fit for the association of age with the respective neuroimaging measures. Shading around the line indicates the SE. The bars underneath the age plots reflect the derivative of the slope, ie, the rate of change taking place at a particular age, scaled as a pseudo $t$ statistic, based on the posterior simulation. CHR-PS+ indicates individuals at clinical high risk who later developed a psychotic disorder; CHR-PS-, individuals at clinical high risk who did not later develop a psychotic disorder. 
general pattern of CT differences observed in individuals with schizophrenia and genetic disorders associated with psychosis and thus suggest that widespread thinner cortex in individuals at CHR may be associated with their increased risk of psychosis.

We also found that lower CT in paracentral, superior temporal, and fusiform regions was associated with psychosis conversion; individuals in the CHR-PS+ group exhibited significantly lower CT than those in the CHR-PS- group and control group in these regions. Lower baseline CT and/or volume in these regions has previously been reported in individuals at CHR who later developed a psychotic disorder ${ }^{17,18}$ (data not used here). Furthermore, longitudinal CT decreases in these regions have been associated with transition to psychosis in those at CHR. ${ }^{6,19,20}$ The magnitude of altered CT in individuals in the CHR-PS+ group in the paracentral, superior temporal, and fusiform regions was highly consistent with findings in individuals with schizophrenia, ${ }^{33,51,52}$ and lower fusiform and paracentral CT has been observed in individuals who hear voices but do not have a diagnosis of schizophrenia. ${ }^{53}$ Given that both helpseeking and non-help-seeking individuals on the psychosis spectrum exhibit alterations in these regions, CT in the paracentral, superior temporal, and fusiform areas may display a dose-response association with psychosis risk. While this interpretation also aligns with our observation that CT in these regions differed between the CHR-PS+, CHR-PS-, and control groups (with the lowest CT for those in the CHR-PS+ group), this explanation remains speculative in light of the cross-sectional nature of the data.

Consistent with previous CHR studies examining baseline neuroimaging associations with later conversion to psychosis, ${ }^{17}$ we did not observe widespread subcortical volume or SA differences associated with later psychosis transition. Taken together, these results suggest that CT reductions may be among the most widespread, robust, and specific morphometric changes associated with psychosis risk and conversion compared with SA or subcortical volume.

An intriguing pattern of findings emerged from the psychosis conversion $\times$ age analyses. Compared with the control group, the CHR-PS+ and CHR-PS- groups exhibited significantly lower paracentral and fusiform region CT among those aged 12 to 16 years. Our analyses investigating ageassociated rates of change (estimated using cross-sectional data) seemed to indicate a steeper decline in slope for those in the control group during this time frame, which reached a plateau in adulthood. However, those in the CHR-PS- group displayed a slower decline, and results in the CHR-PS+ group were indicative of a reduced or delayed rate of change. Relative to the normative timetable in healthy controls, these findings may suggest an accelerated developmental decrease in paracentral and/or fusiform CT in the CHR-PS+ and CHRPS- groups, with the greatest declines occurring in the CHR-PS+ group. If indeed normative CT decreases during adolescence represent a period of specialization (where higher-level systems that contribute to adult outcomes are formed $^{54,55}$ ), lower CT, most apparent in those in the CHRPS+ group, could reflect impairments in optimal specializa- tion. However, these observations are speculative, and the veracity of these patterns will be most accurately captured with longitudinal analyses that encompass a wide age range (eg, early childhood through adulthood).

The neuroanatomic pattern of group differences and ageassociated disruptions observed in individuals at CHR may provide important insights into mechanisms underlying increased risk of psychosis. Preclinical models ${ }^{56,57}$ and recent genome-wide association studies ${ }^{58}$ suggest that genetic variants associated with SA are linked to the regulation of neural progenitor cells during fetal development, while genetic markers associated with CT are associated with regulatory processes in adulthood. Thus, CT differences may be the end result of maladaptive maturation-related mechanisms that occur during postfetal development, including proliferation, synaptic pruning, and/or myelination. ${ }^{59-62}$ Thinner CT, particularly in early adolescence (Figure 3), could reflect abnormal synaptic plasticity or pruning, which have both been implicated in in vitro schizophrenia models. ${ }^{63}$ Although excessive synaptic pruning is one plausible explanation for thinner cortex associated with psychosis transition, recent evidence suggests that intracortical myelination and/or expression of myelin-related genes may be mechanisms of cortical thinning. ${ }^{64,65}$

To better understand neurobiological mechanisms underlying psychosis transition in individuals at CHR, investigations of concomitant measures of CT, macroscale white matter tracts, and intracortical myelination are necessary. Finally, it is also possible that lower CT is not a mechanism of psychosis and can instead be attributed to environmental factors or social determinants associated with psychosis, ${ }^{66,67}$ or that lower CT occurs in response to other possible biological mechanisms underlying psychosis (eg, hypothalamic-pituitary-adrenal stress response ${ }^{68}$ ).

Even if CT reductions in individuals at CHR were robust, effect sizes for between-group differences were nevertheless small to moderate and accounted for approximately $1 \%$ of the variance in comparisons between the CHR-PS+ and CHR-PSgroups. The subtle nature of these morphometric differences underscores the importance of adequate statistical power, achievable only through large-scale multisite collaborations. Consistent with recent work showing that schizophrenia polygenic risk scores only improved differentiation of individuals in the CHR-PS+ group from controls (and not those in the CHR-PS+ group from those in the CHR-PS- group), ${ }^{69}$ we anticipate that baseline, univariate sMRI metrics will have a similar impact on psychosis risk prediction algorithms. Given the logistic and financial challenges that MRI brings, the use of MRI metrics in isolation may not be feasible or useful for psychosis risk prediction. A viable solution may be to adopt sequential assessment frameworks, as recently implemented. ${ }^{70}$ Alternatively, sMRI differences may be a better predictor of general psychopathology and would be better suited for transdiagnostic risk prediction models. ${ }^{71}$

\section{Limitations}

Our study had limitations. One limitation common to multisite studies is that data were collected from multiple scan- 
ners, although leave-1-out analyses suggest that site associations were not prominent. Second, this initial study focused on baseline cross-sectional data and did not investigate progressive sMRI changes associated with psychosis conversion, as identified in prior work. ${ }^{6,18-21,72}$ Additionally, CHR status is associated with heterogeneous outcomes, ${ }^{73-75}$ and neuroimaging phenotypes may differentiate among variability in psychosocial functioning and/or among other psychiatric diagnoses (eg, mood and anxiety disorders). These are two future goals of the ENIGMA Clinical High Risk for Psychosis Working Group, now that feasibility of this collaboration has been established.

\section{Conclusions}

In the largest study of brain abnormalities in individuals at CHR to date, we found robust evidence of a subtle, widespread pattern of CT differences, consistent with observations in psychosis. The specificity of these differences to CT-as well as age-associated deviations in regions sensitive to psychosis conversion-may point to abnormal development processes. These findings also point to age ranges (ie, early adolescence) when morphometric abnormalities in individuals at CHR might be greatest.

\section{ARTICLE INFORMATION}

Accepted for Publication: March 4, 2021.

Published Online: May 5, 2021.

doi:10.1001/jamapsychiatry.2021.0638

ENIGMA Clinical High Risk for Psychosis Working Group Authors: Maria Jalbrzikowski, PhD; Rebecca A. Hayes, PhD; Stephen J. Wood, PhD; Dorte Nordholm, MD, PhD; Juan H. Zhou, PhD; Paolo Fusar-Poli, MD, PhD; Peter J. Uhlhaas, PhD; Tsutomu Takahashi, MD, PhD; Gisela Sugranyes, MD, PhD; Yoo Bin Kwak, BA; Daniel H. Mathalon, MD, PhD; Naoyuki Katagiri, MD, PhD; Christine I. Hooker, PhD; Lukasz Smigielski, PhD; Tiziano Colibazzi, MD; Esther Via, MD, PhD; Jinsong Tang, MD, PhD; Shinsuke Koike, MD, PhD; Paul E. Rasser, MSc; Chantal Michel, PhD; Irina Lebedeva, PhD, DSci; Wenche ten Velden Hegelstad, PhD; Camilo de la Fuente-Sandoval, MD, PhD; James A. Waltz, PhD; Romina Mizrahi, MD, PhD; Cheryl M. Corcoran, MD; Franz Resch, MD; Christian K. Tamnes, PhD; Shalaila S. Haas, PhD; Imke L. J. Lemmers-Jansen, PhD; Ingrid Agartz, MD, PhD; Paul Allen, PhD; G. Paul Amminger, MD, PhD; Ole A. Andreassen, PhD; Kimberley Atkinson, MSc; Peter Bachman, PhD; Inmaculada Baeza, MD, PhD; Helen Baldwin, MSc; Cali F. Bartholomeusz, PhD; Stefan Borgwardt, MD; Sabrina Catalano; Michael W. L. Chee, MBBS Xiaogang Chen, MD, PhD; Kang Ik K. Cho, PhD; Rebecca E. Cooper, BBmed(hons); Vanessa L. Cropley, PhD; Montserrat Dolz, MD; Bjørn H. Ebdrup, MD, PhD; Adriana Fortea, MD; Louise Birkedal Glenthøj, MSc, PhD; Birte Y. Glenthøj, MD, DrMedSci; Lieuwe de Haan, MD, PhD; Holly K. Hamilton, PhD; Mathew A. Harris, PhD; Kristen M. Haut, PhD; Ying He, MD, PhD; Karsten Heekeren, MD, MA; Andreas Heinz, MD, PhD; Daniela Hubl, MD; Wu Jeong Hwang, BBmed(hons); Michael Kaess, MD; Kiyoto Kasai, MD, PhD; Minah Kim, MD, PhD; Jochen Kindler, MD; Mallory J. Klaunig, PhD; Alex Koppel, HBSc; Tina D. Kristensen, PhD; Jun Soo Kwon, MD, PhD; Stephen M. Lawrie, MD(Hons); Jimmy Lee, MBBS, MMed; Pablo León-Ortiz, MD, PhD; Ashleigh Lin, PhD; Rachel L. Loewy, PhD; Xiaoqian Ma, MD; Patrick McGorry, MD, PhD; Philip McGuire, MD, PhD; Masafumi Mizuno, MD, PhD; Paul Møller, MD, PhD; Tomas Moncada-Habib, MD; Daniel Muñoz-Samons, MD; Barnaby Nelson, PhD; Takahiro Nemoto, MD, PhD; Merete Nordentoft, MD, DrMedSci; Maria A. Omelchenko, PhD; Ketil Oppedal, PhD; Lijun Ouyang, MD; Christos Pantelis, MD; Jose C. Pariente, MSc; Jayachandra M. Raghava, PhD; Francisco Reyes-Madrigal, MD, MSc; Brian J. Roach, MS; Jan I. Røssberg, MD, PhD; Wulf Rössler, MD MSc; Dean F. Salisbury, PhD; Daiki Sasabayashi, MD,
PhD; Ulrich Schall, MD, PhD, DSc; Jason Schiffman PhD; Florian Schlagenhauf, MD, PhD; Andre Schmidt, PhD; Mikkel E. Sørensen, MSc; Michio Suzuki, MD, PhD; Anastasia Theodoridou, MD, PhD; Alexander S. Tomyshev, MSc; Jordina Tor, MSc; Tor G. Værnes, MSc; Dennis Velakoulis, MD; Gloria D. Venegoni, MSc; Sophia Vinogradov, MD; Christina Wenneberg, MD, PhD; Lars T. Westlye, PhD; Hidenori Yamasue, MD, PhD; Liu Yuan, MD; Alison R. Yung, PhD; Thérèse A. M. J. van Amelsvoort, MD, PhD; Jessica A. Turner, PhD; Theo G. M. van Erp, PhD; Paul M. Thompson, PhD; Dennis Hernaus, PhD.

\section{Affiliations of ENIGMA Clinical High Risk for} Psychosis Working Group Authors: Department of Psychiatry, University of Pittsburgh, Pittsburgh, Pennsylvania (Jalbrzikowski, Hayes, Bachman, Catalano, Salisbury); Centre for Youth Mental Health, University of Melbourne, Melbourne, Australia (Wood, Amminger, Bartholomeusz, McGorry, Nelson, Yung); Orygen, Melbourne, Australia (Wood, Amminger, Bartholomeusz, McGorry, Nelson, Yung); School of Psychology, University of Birmingham, Birmingham, United Kingdom (Wood); Copenhagen Research Center for Mental Health, Mental Health Center Copenhagen, Copenhagen University Hospital, Copenhagen, Denmark (Nordholm, L. B. Glenthøj, Kristensen, Nordentoft, Wenneberg); Center for Sleep and Cognition, Yong Loo Lin School of Medicine, National University of Singapore, Singapore (Zhou, Chee); Center for Translational Magnetic Resonance Research, Yong Loo Lin School of Medicine, National University of Singapore, Singapore (Zhou); Department of Brain and Behavioral Sciences, University of Pavia, Pavia, Italy (Fusar-Poli); EPIC Lab, Department of Psychosis Studies, King's College London, London, United Kingdom (Fusar-Poli); Department of Child and Adolescent Psychiatry, Charité Universitätsmedizin, Berlin, Germany (Uhlhaas); Institute of Neuroscience and Psychology, University of Glasgow, Glasgow, United Kingdom (Uhlhaas); Department of Neuropsychiatry, University of Toyama Graduate School of Medicine and Pharmaceutical Sciences, Toyama, Japan (Takahashi, Sasabayashi, Suzuki); Research Center for Idling Brain Science, University of Toyama, Toyama, Japan (Takahashi, Sasabayashi, Suzuki); Department of Child and Adolescent Psychiatry and Psychology, Institute of Neuroscience, 2017SGR-881, Hospital Clinic Barcelona, Institut d'Investigacions Biomèdiques August Pi i Sunyer, Centro de Investigación Biomédica en Red de Salud Mental (CIBERSAM), Universitat de Barcelona, Barcelona, Spain (Sugranyes, Baeza); Department of Brain and
Cognitive Sciences, Seoul National University College of Natural Sciences, Seoul, Republic of Korea (Kwak, Hwang); Department of Psychiatry and Behavioral Sciences, University of California, San Francisco (Mathalon, Hamilton, Loewy, Roach); San Francisco Veterans Affairs Health Care System, San Francisco, California (Mathalon, Hamilton, Roach); Department of Neuropsychiatry, Toho University School of Medicine, Tokyo, Japan (Katagiri, Mizuno, Nemoto); Department of Psychiatry and Behavioral Sciences, Rush University Medical Center, Chicago, Illinois (Hooker, Haut); Department of Child and Adolescent Psychiatry, Psychiatric University Hospital Zurich, University of Zurich, Zurich, Switzerland (Smigielski); Department of Psychiatry, Psychotherapy and Psychosomatics, Psychiatric University Hospital Zurich, University of Zurich, Zurich, Switzerland (Smigielski, Heekeren, Rössler, Theodoridou); Department of Psychiatry, Columbia University, New York, New York (Colibazzi); New York State Psychiatric Institute, New York (Colibazzi); Child and Adolescent Mental Health Research Group, Institut de Recerca Sant Joan de Déu, Barcelona, Spain (Via, Dolz, Muñoz-Samons, Tor); Child and Adolescent Psychiatry and Psychology Department, Hospital Sant Joan de Déu, Barcelona, Spain (Via, Dolz, Muñoz-Samons, Tor); Department of Psychiatry, Sir Run Run Shaw Hospital, School of Medicine, Zhejiang University, Hangzhou, China (Tang); Key Laboratory of Medical Neurobiology of Zhejiang Province, School of Medicine, Zhejiang University Hangzhou, Hangzhou, China (Tang); Center for Evolutionary Cognitive Sciences, Graduate School of Art and Sciences, The University of Tokyo, Tokyo, Japan (Koike); The University of Tokyo Institute for Diversity and Adaptation of Human Mind, Tokyo, Japan (Koike, Kasai); Priority Centre for Brain and Mental Health Research, The University of Newcastle, Newcastle, Australia (Rasser, Schall); Priority Research Centre for Stroke and Brain Injury, The University of Newcastle, Newcastle, Australia (Rasser); University Hospital of Child and Adolescent Psychiatry and Psychotherapy, University of Bern, Bern, Switzerland (Michel, Kaess, Kindler); Mental Health Research Center, Moscow, Russia (Lebedeva, Omelchenko, Tomyshev); Faculty of Social Sciences, University of Stavanger, Stavanger, Norway (Hegelstad); TIPS Centre for Clinical Research in Psychosis, Stavanger University Hospital, Stavanger, Norway (Hegelstad); Laboratory of Experimental Psychiatry, Instituto Nacional de Neurología y Neurocirugía, Mexico City, Mexico (de la Fuente-Sandoval, León-Ortiz, Moncada-Habib, Reyes-Madrigal); Maryland 
Psychiatric Research Center, University of Maryland School of Medicine, Baltimore (Waltz); Douglas Research Center, Montreal, Quebec, Canada (Mizrahi); McGill University, Department of Psychiatry, Montreal, Quebec, Canada (Mizrahi); Department of Psychiatry, Icahn School of Medicine at Mount Sinai, New York, New York (Corcoran, Haas); Mental IIIness Research, Education and Clinical Center (MIRECC), James J. Peters VA Medical Center, New York, New York (Corcoran); Clinic for Child and Adolescent Psychiatry, University Hospital of Heidelberg, Heidelberg, Germany (Resch); Department of Psychiatric Research, Diakonhjemmet Hospital, Oslo, Norway (Tamnes, Agartz); NORMENT, Division of Mental Health and Addiction, Oslo University Hospital and Institute of Clinical Medicine, University of Oslo, Oslo, Norway (Tamnes, Agartz, Andreassen, Røssberg, Værnes, Westlye); PROMENTA Research Center, Department of Psychology, University of Oslo, Oslo, Norway (Tamnes); Faculty of Behavioural and Movement Sciences, Department of Clinical, Neuro and Developmental Psychology, Vrije Universiteit Amsterdam, Amsterdam, the Netherlands (Lemmers-Jansen); Department of Clinical Neuroscience, Karolinska Institutet, Stockholm, Sweden (Agartz); Department of Psychology, University of Roehampton, London, United Kingdom (Allen); Department of Psychosis Studies, Institute of Psychiatry, Psychology and Neuroscience, King's College London, London, United Kingdom (Allen, Baldwin, McGuire); Division of Psychiatry, University of Edinburgh, Edinburgh, United Kingdom (Atkinson, Harris, Lawrie, Venegoni); NIHR Maudsley Biomedical Research Centre, South London and Maudsley NHS Foundation Trust and King's College London, London, United Kingdom (Baldwin); Department of Psychiatry, University of Basel, Basel, Switzerland (Borgwardt, Schmidt); Department of Psychiatry and Psychotherapy, University of Lübeck, Lübeck Germany (Borgwardt); National Clinical Research Center for Mental Disorders and Department of Psychiatry, The Second Xiangya Hospital of Central South University, Changsha, China (Chen, He, Ma, Ouyang, Yuan); National Clinical Research Center for Geriatric Disorders, Xiangya Hospital, Central South University, Changsha, China (Chen, Ouyang, Yuan); Department of Psychiatry, Brigham and Women's Hospital, Harvard Medical School, Boston, Massachusetts (Cho); Melbourne Neuropsychiatry Centre, Department of Psychiatry, University of Melbourne and Melbourne Health, Melbourne, Australia (Cooper, Cropley, Pantelis, Velakoulis); Centre for Mental Health, Faculty of Health, Arts and Design, School of Health Sciences, Swinburne University, Melbourne, Australia (Cropley, Pantelis); Center for Clinical Intervention and Neuropsychiatric Schizophrenia Research, Mental Health Centre Glostrup, University of Copenhagen, Glostrup, Denmark (Ebdrup, B. Y. Glenthøj, Kristensen, Raghava, Sørensen); Department of Clinical Medicine, Faculty of Health and Medical Sciences, University of Copenhagen, Copenhagen, Denmark (Ebdrup, B. Y. Glenthøj); Department of Child and Adolescent Psychiatry and Psychology, Institute of Neuroscience, Hospital Clinic Barcelona, Fundació Clínic Recerca Biomèdica, Universitat de Barcelona, Barcelona, Spain (Fortea); Department of Psychiatry, Amsterdam University Medical Centre, Amsterdam, the Netherlands (de Haan); Arkin, Amsterdam, the Netherlands (de Haan); Department of Psychiatry and Psychotherapy I,
LVR-Hospital Cologne, Cologne, Germany (Heekeren); Department of Psychiatry and Psychotherapy, Charité Universitätsmedizin, Berlin, Germany (Heinz, Rössler, Schlagenhauf); Translational Research Center, University Hospital of Psychiatry and Psychotherapy, University of Bern, Bern, Switzerland (Hubl); Department of Child and Adolescent Psychiatry, Center of Psychosocial Medicine, University of Heidelberg, Heidelberg, Germany (Kaess); Department of Neuropsychiatry, Graduate School of Medicine, The University of Tokyo, Tokyo, Japan (Kasai); The International Research Center for Neurointelligence at The University of Tokyo Institutes for Advanced Study, The University of Tokyo, Tokyo, Japan (Kasai); Department of Neuropsychiatry, Seoul National University Hospital, Seoul, Republic of Korea (Kim, Kwon); Department of Psychiatry, Seoul National University College of Medicine, Seoul, Republic of Korea (Kim, Kwon); Department of Psychology, University of Maryland, Baltimore County, Baltimore (Klaunig, Schiffman); Department of Pharmacology and Toxicology, University of Toronto, Toronto, Ontario, Canada (Koppel): Department of Psychosis, Institute of Mental Health, Singapore (Lee); Lee Kong Chian School of Medicine, Nanyang Technological University, Singapore (Lee); Telethon Kids Institute, The University of Western Australia, Perth, Australia (Lin); Department for Mental Health Research and Development, Division of Mental Health and Addiction, Vestre Viken Hospital Trust, Lier, Norway (Møller); Stavanger Medical Imaging Laboratory, Department of Radiology, Stavanger University Hospital, Stavanger, Norway (Oppedal); Hunan Key Laboratory of Psychiatry and Mental Health, Changsha, China (Ouyang, Yuan); Florey Institute of Neuroscience and Mental Health, University of Melbourne, Melbourne, Australia (Pantelis); Magnetic Resonance Imaging Core Facility, Institut d'Investigacions Biomèdiques August Pi i Sunyer Barcelona, Spain (Pariente); Department of Clinica Physiology, Nuclear Medicine and PET, Functional Imaging Unit, University of Copenhagen, Glostrup, Denmark (Raghava); Priority Research Centre Grow Up Well, The University of Newcastle, Newcastle, Australia (Schall); Department of Psychological Science, University of California, Irvine (Schiffman); Early Intervention in Psychosis Advisory Unit for South-East Norway, TIPS Sør-Øst, Division of Mental Health and Addiction, Oslo University Hospital, Oslo, Norway (Værnes); Neuropsychiatry, The Royal Melbourne Hospital, Melbourne, Australia (Velakoulis); Department of Psychiatry and Neuropsychology, School for Mental Health and Neuroscience, Faculty of Health Medicine and Life Sciences, Maastricht University, Maastricht, the Netherlands (Venegoni, van Amelsvoort, Hernaus); Department of Psychiatry and Behavioral Sciences, University of Minnesota, Minneapolis (Vinogradov); Department of Psychology, University of Oslo, Oslo, Norway (Westlye); Department of Psychiatry, Hamamatsu University School of Medicine, Hamamatsu City, Japan (Yamasue); School of Health Sciences, University of Manchester, Manchester, United Kingdom (Yung); Department of Psychology, Georgia State University, Atlanta (Turner); Center for the Neurobiology of Learning and Memory, Irvine, California (van Erp); Clinical Translational Neuroscience Laboratory, Department of Psychiatry and Human Behavior, University of
California, Irvine (van Erp); Imaging Genetics Center, Mark and Mary Stevens Institute for Neuroimaging and Informatics, Keck School of Medicine of USC, University of Southern California, Los Angeles (Thompson).

Author Contributions: Drs Jalbrzikowski and Hernaus had full access to all of the data in the study and take responsibility for the integrity of the data and the accuracy of the data analysis. Study concept and design: Jalbrzikowski, Hernaus. Acquisition, analysis, or interpretation of data: All authors. Drafting of the manuscript: Jalbrzikowski, Hernaus. Critical revision of the manuscript for important intellectual content: All authors.

Statistical analysis: Jalbrzikowski, Hernaus. Administrative, technical, or material support: Jalbrzikowski, Hayes, Catalano, Hernaus. Study supervision: Jalbrzikowski, Wood, Nordholm, Zhou, Fusar-Poli, Uhlhaas, Takahashi, Sugranyes, Kwak, Mathalon, Katagiri, Hooker, Smigielski, Colibazzi, Via, Tang, Koike, Michel, Lebedeva, Hegelstad, de la Fuente-Sandoval, Waltz, Mizrahi, Corcoran, Resch, Tamnes, Lemmers-Jansen, Amminger, Dolz, van Amelsvoort, Turner, van Erp, Thompson, Hernaus.

Conflict of Interest Disclosures: Dr Fusar-Poli has received grants from Lundbeck; personal fees from Angelini Pharma and Menarini Group; and nonfinancial support from Boehringer Ingelheim. Dr Uhlhaas has received grants from Lundbeck and Eli Lilly UK. Dr Takahashi has received personal fees from Toyama Hospital, Taikoyama Hospital, Saiseikai Toyama Hospital, and Greenhills Wakakusa Hospital. Dr Mathalon has served as a consultant to Boehringer Ingelheim, Cadent Therapeutics, Syndesi Therapeutics, and Recognify. Drs Via and Dolz have received personal fees from Janssen-Cilag. Dr Koike has received grants from Agency for Medical Research and Development, Japan Society for the Promotion of Science, the Naito Foundation, and Takeda Science Foundation. Dr Andreassen has received personal fees from Lundbeck and Sunovion and has served as a consultant for HealthLytix. Dr Baeza has received personal fees from Angelini Pharma, Janssen Pharmaceuticals, and Otsuka-Lundbeck. Dr Chee has served as a sleep consultant for the AIA Vitality Program. Dr Ebdrup has received personal fees for serving on advisory boards from Eli Lilly Denmark, Janssen-Cilag, Lundbeck, and Takeda and has received lecture fees from Bristol Myers Squibb, Otsuka Pharma Scandinavia, Eli Lilly and Company, Boehringer Ingelheim Denmark, and Lundbeck. Dr Fortea has received honoraria from Otsuka-Lundbeck. Dr Birte Glenthøj is the leader of a Lundbeck Foundation Centre of Excellence for Clinical Intervention and Neuropsychiatric Schizophrenia Research, which is partially funded by an independent grant from the Lundbeck Foundation and partially funded by the Mental Health Services in the Capital Region of Denmark, the University of Copenhagen, and other foundations. Dr Kaess has received grants from German Federal Ministry of Education and Research, Swiss National Science Foundation, German Research Foundation, Dietmar Hopp Foundation, Baden-Wuerttemberg Foundation, and National Health, Medical and Research Council. Dr Kasai has received grants from Novartis, Astellas, Merck Sharp and Dohme, Eli Lily and Company, Dainippon-Sumitomo Corporation, Eisai, Otsuka, Shionogi, Ono, Tanabe-Mitsubishi, and Takeda; 
personal fees from Otsuka, Fuji-Film, Yoshitomi, Kyowa, Janssen Pharmaceuticals, Astellas, Meiji Seika Pharma, Sumitomo Dainippon Pharma, and Takeda; and serves on the funding committees of Takeda Science Foundation and Astellas. Dr Kindler has received grants from the Swiss National Science Foundation. Dr Kristensen has received grants from Lundbeck Foundation. Dr Lawrie has received personal fees from Janssen Pharmaceuticals and Sunovion. Dr León-Ortiz has received personal fees from Janssen Pharmaceuticals. Dr Loewy has received grants from National Institute of Mental Health and California Mental Health Services Authority. Dr Nemoto has received grants from Otsuka as well as personal fees from Astellas, Eisai, Janssen Pharmaceuticals, Meiji Seika Pharma, Sumitomo Dainippon Pharma, and Takeda. Dr Pantelis has received grants and personal fees from Lundbeck Foundation. Dr Reyes-Madrigal has received speaker fees from Janssen (Johnson \& Johnson). Dr Rössler is supported by the Zurich Program for Sustainable Development of Mental Health Services. Dr Suzuki had received personal fees from Nakagawa Hospital. Ms Tor has received personal fees from Fundación Alicia Koplowitz. Dr Vinogradov served as a member of an advisory board for Alkermes and is an unpaid scientific collaborator for PositScience Inc. Dr Hernaus has received consulting fees from P1vital Products. No other disclosures were reported.

Funding/Support: Dr Jalbrzikowski has received grant K01 MH112774 from the National Institute of Mental Health. Dr Hayes has received grants from the University of Pittsburgh Medical Center. Drs Wood, Amminger, Bartholomeusz, McGorry, Nelson, and Yung have received funding from the Colonial Foundation. Drs Wood, McGorry, and Yung have received grant 566529 from the National Health and Medical Research Council. Dr Wood has received Clinical Career Development Award 359223 from the National Health and Medical Research Council. Dr Nordholm has received grants R25-A2701 and R287-2018-1485 from the Lundbeck Foundation and grants from the Mental Health Services Capital Region of Denmark. Dr Zhou has received grants from the Yong Loo Lin School of Medicine at the National University of Singapore. Drs Fusar-Poli, Allen, and McGuire and have received grant UK G0700995 from the UK Medical Research Council. Dr Uhlhaas has received grant MR/L011689/1 from the UK Medical Research Council. Dr Sugranyes has received Young Investigator Award grant 26731 from the Brain and Behavior Research Foundation, grants from the Spanish I+D+I state program, grants from the ISCIII-Subdirección General de Evaluación, grant PI18/00976 from the European Regional Development Fund, grant SLT006/17/00362 from the Government of Catalonia (Programa PERIS Salut Mental), grants from the Alicia Koplowitz Foundation, and grants from the Fundació Clínic Recerca Biomèdica (Ajut a la Recerca Pons Bartran). Dr Mathalon has received grants from the Brain and Behavior Research Foundation and grant R01 MH076989 from the National Institute of Mental Health. Dr Hooker was supported by grant R01 MH105246 from the National Institutes of Health. Dr Colibazzi has received grants 5T32MH15144 and K23 MH85063 from the National Institute of Mental Health as well as grants from the Brain and Behavior Research Foundation, Sackler Institute, Herbert Irving Scholar Award, and Columbia University Bodini Fellowship. Dr Tang has received grant 81871057 from the National Natural Science Foundation of China. Drs Koike and Kasai have received grants JP20dm0307004 and JP20dm0207069 from the Japan Agency for Medical Research and Development. Dr Koike has received grant JP18K07550 from the Japan Society for the Promotion of Science KAKENHI. Dr Lebedeva and Mr Tomyshev were supported by grant 20-013-00748 from the Russian Foundation for Basic Research. Dr de la Fuente-Sandoval was supported by grants 182279 and 261895 from the Consejo Nacional de Ciencia y Tecnología, grants from CONACYT's Sistema Nacional de Investigadores, and grant R21 MH117434 from the National Institutes of Health. Dr Waltz has received grant 5R01MH115031 from the National Institute of Mental Health. Dr Mizrahi has received grants R01MH100043 and R01MH113564 from the National Institute of Mental Health and grants from the Brain and Behavior Research Foundation and Canadian Institutes of Health Research. Dr Corcoran has received grant $\mathrm{R} 01 \mathrm{MH} 107558$ from the National Institutes of Health. Dr Tamnes has received grants 288083 and 223273 from the Research Council of Norway and grant 2019069 from the South-Eastern Norway Regional Health Authority. Dr Amminger has received Senior Research Fellowship grant 1080963 from the National Health and Medical Research Council. Dr Andreassen has received grants 223273 and 283798 from the Research Council of Norway as well as grants from K. G. Jebsen Stiftelsen and UiO Life Science. Ms Atkinson has received grants from the University of Edinburgh. Dr Baeza has received grants INT19/00021, PI11/1349, PI15/0444, and PI180242 from the Instituto de Salud Carlos III. Ms Baldwin has received funding from the National Institute for Health Research Maudsley Biomedical Research Centre studentship. Dr Borgwardt has received grants from the SYNSCHIZ project under the ERA-NET Horizon 2020 program. Drs Chee and Lee received grant NMRC/TCR/003/2008 from the National Medical Research Council Translational and Clinical Research Flagship Programme. Dr Chee has received grant NMRC/STaR/0015/2013 from the National Medical Research Council. Dr Cropley has received investigator grant 1177370 and project grant 1065742 from the National Health and Medical Research Council. Dr Dolz has received grants PI11/02684 and PI15/00509 from the Instituto de Salud Carlos III and a grant from the Alicia Koplowitz Foundation. Dr Louise Glenthøj was supported by the Tryg Foundation and Danish Research Council. Dr Birte Glenthøj was supported by the Lundbeck Foundation Center for Clinical Intervention and Neuropsychiatric Schizophrenia Research. Mr Harris has received grants from the Wellcome Trust. Dr Kasai has received grants JP18dm0207004 and JP2Odm0307001 from the Japan Agency for Medical Research and Development and grants JP16H06395, JP16H06399, JP16K21720, JP16H06280, and $20 \mathrm{HO} 3596$ from the Japan Society for the Promotion of Science KAKENHI. Dr Klaunig has received grants from the National Institute of Mental Health. Dr Kwon has received grant 2017M3C7A1029610 from the National Research Foundation of Korea. Dr Lin was supported by grant 1148793 from the Australian National Health and Medical Research Council. Dr Loewy has received grants K23MH086618 and R01MH081051 from the National Institute of Mental Health. Drs McGorry and Yung have received grant 350241 from the
National Health and Medical Research Council. Dr Nelson has received Career Development Fellowship grant 1027532, Senior Research Fellowship grant 1137687, and project grant 1027741 from the National Health and Medical Research Council. Dr Oppedal has received postdoctoral scholarship grant 912152 from the Western Norway Regional Health Authority. Dr Pantelis has received Senior Principal Research Fellowship grant 1105825 and L3 Investigator grant 1196508 from the National Health and Medical Research Council as well as Distinguished Investigator Award 18722 from the Brain and Behavior Research Foundation. Dr Rössler has received grants from the Zurich Program for Sustainable Development of Mental Health Services. Dr Salisbury has received grant R01 MH113533 from the National Institute of Mental Health. Dr Sasabayashi has received grant JP18K15509 from the Japan Society for the Promotion of Science KAKENHI. Dr Schall has received grant 569259 from the National Health and Medical Research Council of Australia. Dr Schiffman has received grants from the Maryland Department of Health and Mental Hygiene, Behavioral Health Administration through the Maryland Center of Excellence on Early Intervention Program, grant 14-13717G/ MOOB4400241 from the Office of Procurement and Support Services, and grant RO1MH112612 from the National Institute of Mental Health. Dr Suzuki has received grant JP2OH03598 from the Japan Society for the Promotion of Science KAKENHI and grant JP19dk0307069s0203 from the Japan Agency for Medical Research and Development. Ms Tor has received grants PI11/02684 and PI15/ 00509 from the Instituto de Salud Carlos III. Dr Westlye has received grant 300767 from the Research Council of Norway, grant 2019101 from the South-Eastern Norway Regional Health Authority, and European Union's Horizon 2020 Research and Innovation Program grant 802998 from the European Research Council. Dr Yung has received Principal Research Fellowship grant GNT1136829 from the National Health and Medical Research Council and Senior Research Fellowship grant 566593 from the National Health and Medical Research Council. Dr Turner has received grant 5R01MH094524 from the National Institutes of Health.

Role of the Funder/Sponsor: The funders had no role in the design and conduct of the study; collection, management, analysis, and interpretation of the data; preparation, review, or approval of the manuscript; and decision to submit the manuscript for publication.

\section{REFERENCES}

1. Yung $A R$, McGorry PD, McFarlane CA Jackson HJ, Patton GC, Rakkar A. Monitoring and care of young people at incipient risk of psychosis. Schizophr Bull. 1996;22(2):283-303. doi:10.1093/ schbul/22.2.283

2. Fusar-Poli P, Bonoldi I, Yung AR, et al. Predicting psychosis: meta-analysis of transition outcomes in individuals at high clinical risk. Arch Gen Psychiatry. 2012;69(3):220-229. doi:10.1001/archgenpsychiatry 2011.1472

3. Hartmann JA, Yuen HP, McGorry PD, et al. Declining transition rates to psychotic disorder in "ultra-high risk" clients: investigation of a dilution effect. Schizophr Res. 2016;170(1):130-136. doi:10 1016/j.schres.2015.11.026 
4. Zikidi K, Gajwani R, Gross J, et al. Grey-matter abnormalities in clinical high-risk participants for psychosis. Schizophr Res. 2020;226:120-128. doi:10.1016/j.schres.2019.08.034

5. Velakoulis D, Wood SJ, Wong MTH, et al. Hippocampal and amygdala volumes according to psychosis stage and diagnosis: a magnetic resonance imaging study of chronic schizophrenia, first-episode psychosis, and ultra-high-risk individuals. Arch Gen Psychiatry. 2006;63(2): 139-149. doi:10.1001/archpsyc.63.2.139 6. Sun D, Phillips L, Velakoulis D, et al. Progressive brain structural changes mapped as psychosis develops in 'at risk' individuals. Schizophr Res. 2009;108(1-3):85-92. doi:10.1016/j.schres.2008. 11.026

7. Fornito A, Yung AR, Wood SJ, et al. Anatomic abnormalities of the anterior cingulate cortex before psychosis onset: an MRI study of ultra-high-risk individuals. Biol Psychiatry. 2008;64 (9):758-765. doi:10.1016/j.biopsych.2008.05.032

8. Tomyshev AS, Lebedeva IS, Akhadov TA, Omelchenko MA, Rumyantsev AO, Kaleda VG. Alterations in white matter microstructure and cortical thickness in individuals at ultra-high risk of psychosis: a multimodal tractography and surface-based morphometry study. Psychiatry Res Neuroimaging. 2019;289:26-36. doi:10.1016/j. pscychresns.2019.05.002

9. Kwak YB, Kim M, Cho KIK, Lee J, Lee TY, Kwon JS. Reduced cortical thickness in subjects at clinical high risk for psychosis and clinical attributes. Aust N Z J Psychiatry. 2019;53(3):219-227. doi:10. 1177/0004867418807299

10. Iwashiro N, Suga M, Takano Y, et al. Localized gray matter volume reductions in the pars triangularis of the inferior frontal gyrus in individuals at clinical high-risk for psychosis and first episode for schizophrenia. Schizophr Res. 2012 137(1-3):124-131. doi:10.1016/j.schres.2012.02.024

11. Takayanagi Y, Kulason S, Sasabayashi D, et al. Reduced thickness of the anterior cingulate cortex in individuals with an at-risk mental state who later develop psychosis. Schizophr Bull. 2017;43(4): 907-913. doi:10.1093/schbul/sbw167

12. Chung Y, Allswede D, Addington J, et al; North American Prodrome Longitudinal Study (NAPLS) Consortium. Cortical abnormalities in youth at clinical high-risk for psychosis: findings from the NAPLS2 cohort. Neuroimage Clin. 2019;23:101862. doi:10.1016/j.nicl.2019.101862

13. Koutsouleris N, Meisenzahl EM, Davatzikos C, et al. Use of neuroanatomical pattern classification to identify subjects in at-risk mental states of psychosis and predict disease transition. Arch Gen Psychiatry. 2009;66(7):700-712. doi:10.1001/ archgenpsychiatry.2009.62

14. Klauser P, Zhou J, Lim JKW, et al. Lack of evidence for regional brain volume or cortical thickness abnormalities in youths at clinical high risk for psychosis: findings from the Longitudinal Youth at Risk study. Schizophr Bull. 2015;41(6): 1285-1293. doi:10.1093/schbul/sbv012

15. Ziermans TB, Durston S, Sprong M, et al. No evidence for structural brain changes in young adolescents at ultra high risk for psychosis. Schizophr Res. 2009;112(1-3):1-6. doi:10.1016/j. schres.2009.04.013

16. Fusar-Poli P, Borgwardt $S$, Crescini A, et al. Neuroanatomy of vulnerability to psychosis: a voxel-based meta-analysis. Neurosci Biobehav Rev 2011;35(5):1175-1185. doi:10.1016/j.neubiorev.2010. 12.005
17. Del Re EC, Stone WS, Bouix S, et al. Baseline cortical thickness reductions in clinical high risk for psychosis: brain regions associated with conversion to psychosis versus non-conversion as assessed at one-year follow-up in the Shanghai-At-Risk-forPsychosis (SHARP) study. Schizophr Bull. 2021;47 (2):562-574. doi:10.1093/schbul/sbaa127

18. Borgwardt SJ, McGuire PK, Aston J, et al. Structural brain abnormalities in individuals with an at-risk mental state who later develop psychosis. Br J Psychiatry Suppl. 2007;51:s69-s75. doi:10.1192/ bjp.191.51.s69

19. Pantelis C, Velakoulis D, McGorry PD, et al. Neuroanatomical abnormalities before and after onset of psychosis: a cross-sectional and longitudinal MRI comparison. Lancet. 2003;361 (9354):281-288. doi:10.1016/S0140-6736(03)12323-9

20. Takahashi T, Wood SJ, Yung AR, et al. Progressive gray matter reduction of the superior temporal gyrus during transition to psychosis. Arch Gen Psychiatry. 2009;66(4):366-376. doi:10. 1001/archgenpsychiatry.2009.12

21. Cannon TD, Chung Y, He G, et al; North American Prodrome Longitudinal Study Consortium. Progressive reduction in cortical thickness as psychosis develops: a multisite longitudinal neuroimaging study of youth at elevated clinical risk. Biol Psychiatry. 2015;77(2): 147-157. doi:10.1016/j.biopsych.2014.05.023

22. Sakuma A, Obara C, Katsura M, et al. No regional gray matter volume reduction observed in young Japanese people at ultra-high risk for psychosis: a voxel-based morphometry study. Asian J Psychiatr. 2018;37:167-171. doi:10.1016/j.ajp.2018. 09.009

23. Mechelli A, Riecher-Rössler A, Meisenzahl EM, et al. Neuroanatomical abnormalities that predate the onset of psychosis: a multicenter study. Arch Gen Psychiatry. 2011;68(5):489-495. doi:10.1001/ archgenpsychiatry.2011.42

24. Farris MS, Devoe DJ, Addington J. Attrition rates in trials for adolescents and young adults at clinical high-risk for psychosis: a systematic review and meta-analysis. Early Interv Psychiatry. 2020;14 (5):515-527. doi:10.1111/eip.12864

25. Simon AE, Borgwardt S, Riecher-Rössler A, Velthorst E, de Haan L, Fusar-Poli P. Moving beyond transition outcomes: meta-analysis of remission rates in individuals at high clinical risk for psychosis. Psychiatry Res. 2013;209(3):266-272. doi:10.1016/j. psychres.2013.03.004

26. Button KS, loannidis JPA, Mokrysz C, et al Power failure: why small sample size undermines the reliability of neuroscience. Nat Rev Neurosci. 2013;14(5):365-376. doi:10.1038/nrn3475

27. Jones $P$, Rodgers $B$, Murray R, Marmot M. Child development risk factors for adult schizophrenia in the British 1946 birth cohort. Lancet. 1994;344(8934):1398-1402. doi:10.1016/S01406736(94)90569-X

28. Lauronen E, Miettunen J, Veijola J, Karhu M, Jones PB, Isohanni M. Outcome and its predictors in schizophrenia within the Northern Finland 1966 Birth Cohort. Eur Psychiatry. 2007;22(2):129-136. doi:10.1016/j.eurpsy.2006.07.001

29. Vijayakumar N, Allen NB, Youssef G, et al Brain development during adolescence: a mixed-longitudinal investigation of cortical thickness, surface area, and volume. Hum Brain Mapp. 2016;37(6):2027-2038. doi:10.1002/hbm.23154

30. Tamnes $C K$, Herting MM, Goddings $A-L$, et al. Development of the cerebral cortex across adolescence: a multisample study of inter-related longitudinal changes in cortical volume, surface area, and thickness. J Neurosci. 2017;37(12): 3402-3412. doi:10.1523/JNEUROSCI.3302-16.2017 31. Schimmelmann BG, Michel C, Martz-Irngartinger A, Linder C, Schultze-Lutter F. Age matters in the prevalence and clinical significance of ultra-high-risk for psychosis symptoms and criteria in the general population: findings from the BEAR and BEARS-Kid studies. World Psychiatry. 2015;14(2):189-197. doi:10.1002/ wps.20216

32. van Erp TGM, Hibar DP, Rasmussen JM, et al. Subcortical brain volume abnormalities in 2028 individuals with schizophrenia and 2540 healthy controls via the ENIGMA Consortium. Mol Psychiatry. 2016;21(4):547-553. doi:10.1038/mp.2015.63

33. van Erp TGM, Walton $\mathrm{E}$, Hibar DP, et al; Karolinska Schizophrenia Project. Cortical brain abnormalities in 4474 individuals with schizophrenia and 5098 control subjects via the Enhancing Neuro Imaging Genetics Through Meta Analysis (ENIGMA) Consortium. Biol Psychiatry. 2018;84(9):644-654. doi:10.1016/j.biopsych.2018. 04.023

34. Ching CRK, Gutman BA, Sun D, et al. Mapping subcortical brain alterations in 22q11.2 deletion syndrome: effects of deletion size and convergence with idiopathic neuropsychiatric illness. Am J Psychiatry. 2020;177(7):589-600. doi:10.1176/appi. ajp.2019.19060583

35. Sun $D$, Ching CRK, Lin A, et al. Large-scale mapping of cortical alterations in 22q11.2 deletion syndrome: convergence with idiopathic psychosis and effects of deletion size. Mol Psychiatry. 2020;25(8):1822-1834. doi:10.1038/s41380-018 0078-5

36. World Medical Association. World Medical Association Declaration of Helsinki: ethical principles for medical research involving human subjects. JAMA. 2013;310(20):2191-2194. doi:10. 1001/jama.2013.281053

37. Fischl B, Sereno MI, Dale AM. Cortical surface-based analysis. II: inflation, flattening, and a surface-based coordinate system. Neuroimage. 1999;9(2):195-207. doi:10.1006/nimg.1998.0396

38. Fischl B, Dale AM. Measuring the thickness of the human cerebral cortex from magnetic resonance images. Proc Natl Acad Sci U S A. 2000; 97(20):11050-11055. doi:10.1073/pnas.200033797

39. Fischl B, Salat DH, Busa E, et al. Whole brain segmentation: automated labeling of neuroanatomical structures in the human brain. Neuron. 2002;33(3):341-355. doi:10.1016/S0896 6273(02)00569-X

40. Schmaal L, Veltman DJ, van Erp TGM, et al. Subcortical brain alterations in major depressive disorder: findings from the ENIGMA Major Depressive Disorder Working Group. Mol Psychiatry. 2016;21(6):806-812. doi:10.1038/mp.2015.69

41. Schmaal L, Hibar DP, Sämann PG, et al. Cortical abnormalities in adults and adolescents with major depression based on brain scans from 20 cohorts worldwide in the ENIGMA Major Depressive Disorder Working Group. Mol Psychiatry. 2017;22 (6):900-909. doi:10.1038/mp.2016.60

42. Benjamini Y, Hochberg Y. Controlling the false discovery rate: a practical and powerful approach to multiple testing. J Royal Stat Soc B (Methodological) .1995;57(1):289-300.

43. Radua J, Vieta E, Shinohara R, et al; ENIGMA Consortium Collaborators. Increased power by harmonizing structural MRI site differences with the 
ComBat batch adjustment method in ENIGMA. Neuroimage. 2020;218:116956. doi:10.1016/j. neuroimage.2020.116956

44. Efron B, Stein C. The jackknife estimate of variance. Ann Statist. 1981;9(3):586-596. doi:10. 1214/aos/1176345462

45. Lakens D. Equivalence tests: a practical primer for $t$ tests, correlations, and meta-analyses. Soc Psychol Personal Sci. 2017;8(4):355-362. doi:10. 1177/1948550617697177

46. Wood SN. Generalized Additive Models: An Introduction With R. 2nd ed. CRC Press; 2017. doi:10.1201/9781315370279

47. Hastie T, Tibshirani R. Generalized additive models. Statist Sci. 1986;1(3):297-310. doi:10.1214/ ss/1177013604

48. Jalbrzikowski M, Larsen $B$, Hallquist MN, Foran W, Calabro F, Luna B. Development of white matter microstructure and intrinsic functional connectivity between the amygdala and ventromedial prefrontal cortex: associations with anxiety and depression. Biol Psychiatry. 2017;82(7): 511-521. doi:10.1016/j.biopsych.2017.01.008

49. Marek S, Hwang $K$, Foran W, Hallquist MN, Luna B. The contribution of network organization and integration to the development of cognitive control. PLoS Biol. 2015;13(12):e1002328. doi:10. 1371/journal.pbio.1002328

50. Writing Committee for the Attention-Deficit/ Hyperactivity Disorder, Autism Spectrum Disorder, Bipolar Disorder, Major Depressive Disorder, Obsessive-Compulsive Disorder, and Schizophrenia ENIGMA Working Groups. Virtual histology of cortical thickness and shared neurobiology in 6 psychiatric disorders. JAMA Psychiatry. 2021;78(1): 47-63. doi:10.1001/jamapsychiatry.2020.2694

51. Onitsuka T, Shenton ME, Kasai K, et al. Fusiform gyrus volume reduction and facial recognition in chronic schizophrenia. Arch Gen Psychiatry. 2003; 60(4):349-355. doi:10.1001/archpsyc.60.4.349

52. Takahashi T, Suzuki M, Zhou S-Y, et al. Temporal lobe gray matter in schizophrenia spectrum: a volumetric MRI study of the fusiform gyrus, parahippocampal gyrus, and middle and inferior temporal gyri. Schizophr Res. 2006;87(1-3):116-126. doi:10.1016/j.schres.2006.04.023

53. van Lutterveld $R$, van den Heuvel MP, Diederen KMJ, et al. Cortical thickness in individuals with non-clinical and clinical psychotic symptoms. Brain. 2014;137(pt 10):2664-2669. doi:10.1093/brain/ awu167

54. Murty VP, Calabro F, Luna B. The role of experience in adolescent cognitive development: integration of executive, memory, and mesolimbic systems. Neurosci Biobehav Rev. 2016;70:46-58. doi:10.1016/j.neubiorev.2016.07.034
55. Calabro FJ, Murty VP, Jalbrzikowski M, Tervo-Clemmens B, Luna B. Development of hippocampal-prefrontal cortex interactions through adolescence. Cereb Cortex. 2020;30(3): 1548-1558. doi:10.1093/cercor/bhz186

56. Pontious A, Kowalczyk T, Englund C, Hevner RF. Role of intermediate progenitor cells in cerebral cortex development. Dev Neurosci. 2008;30(1-3): 24-32. doi:10.1159/000109848

57. Rakic P. Specification of cerebral cortical areas. Science. 1988;241(4862):170-176. doi:10.1126/science. 3291116

58. Grasby KL, Jahanshad N, Painter JN, et al; Alzheimer's Disease Neuroimaging Initiative; CHARGE Consortium; EPIGEN Consortium; IMAGEN Consortium; SYS Consortium; Parkinson's Progression Markers Initiative; Enhancing Neurolmaging Genetics through Meta-Analysis Consortium (ENIGMA) - Genetics working group. The genetic architecture of the human cerebral cortex. Science. 2020;367(6484):eaay6690. doi:10.1126/science.aay6690

59. Huttenlocher PR. Synaptic density in human frontal cortex-developmental changes and effects of aging. Brain Res. 1979;163(2):195-205. doi:10. 1016/0006-8993(79)90349-4

60. Rakic $P$, Bourgeois JP, Eckenhoff MF, Zecevic N, Goldman-Rakic PS. Concurrent overproduction of synapses in diverse regions of the primate cerebra cortex. Science. 1986;232(4747):232-235. doi:10. 1126/science.3952506

61. Petanjek Z, Judas M, Kostović I, Uylings HBM. Lifespan alterations of basal dendritic trees of pyramidal neurons in the human prefrontal cortex: a layer-specific pattern. Cereb Cortex. 2008;18(4): 915-929. doi:10.1093/cercor/bhm124

62. Natu VS, Gomez J, Barnett M, et al. Apparent thinning of human visual cortex during childhood is associated with myelination. Proc Natl Acad Sci USA. 2019;116(41):20750-20759. doi:10.1073/pnas. 1904931116

63. Sellgren CM, Gracias J, Watmuff B, et al. Increased synapse elimination by microglia in schizophrenia patient-derived models of synaptic pruning. Nat Neurosci. 2019;22(3):374-385. doi:10. 1038/s41593-018-0334-7

64. Whitaker KJ, Vértes PE, Romero-Garcia R, et al; NSPN Consortium. Adolescence is associated with genomically patterned consolidation of the hubs of the human brain connectome. Proc Natl Acad Sci U S A. 2016;113(32):9105-9110. doi:10.1073/pnas. 1601745113

65. Parker N, Patel Y, Jackowski AP, et al; Saguenay Youth Study and the IMAGEN Consortium. Assessment of neurobiological mechanisms of cortical thinning during childhood and adolescence and their implications for psychiatric disorders.
JAMA Psychiatry. 2020;77(11):1127-1136. doi:10 1001/jamapsychiatry.2020.1495

66. Murray RM, Mondelli V, Stilo SA, et al. The influence of risk factors on the onset and outcome of psychosis: what we learned from the GAP study. Schizophr Res. 2020;225:63-68. doi:10. 1016/j.schres.2020.01.011

67. Anglin DM, Galea S, Bachman P. Going upstream to advance psychosis prevention and improve public health. JAMA Psychiatry. 2020;77 (7):665-666. doi:10.1001/jamapsychiatry.2020.0142

68. Pruessner M, Cullen AE, Aas M, Walker EF. The neural diathesis-stress model of schizophrenia revisited: an update on recent findings considering illness stage and neurobiological and

methodological complexities. Neurosci Biobehav Rev. 2017;73:191-218. doi:10.1016/j.neubiorev.2016.12.013

69. Perkins DO, Olde Loohuis L, Barbee J, et al. Polygenic risk score contribution to psychosis prediction in a target population of persons at clinical high risk. Am J Psychiatry. 2020;177(2): 155-163. doi:10.1176/appi.ajp.2019.18060721

70. Koutsouleris N, Kambeitz-Ilankovic L, Ruhrmann S, et al: PRONIA Consortium. Prediction models of functional outcomes for individuals in the clinical high-risk state for psychosis or with recent-onset depression: a multimodal, multisite machine learning analysis. JAMA Psychiatry. 2018; 75(11):1156-1172. doi:10.1001/jamapsychiatry. 2018.2165

71. McGorry PD, Hartmann JA, Spooner R, Nelson B. Beyond the "at risk mental state" concept: transitioning to transdiagnostic psychiatry. World Psychiatry. 2018;17(2):133-142. doi:10.1002/ wps.20514

72. Dazzan P, Soulsby B, Mechelli A, et al. Volumetric abnormalities predating the onset of schizophrenia and affective psychoses: an MRI study in subjects at ultrahigh risk of psychosis. Schizophr Bull. 2012;38(5):1083-1091. doi:10.1093/ schbul/sbrO35

73. Rutigliano G, Valmaggia $L$, Landi $P$, et al. Persistence or recurrence of non-psychotic comorbid mental disorders associated with 6-year poor functional outcomes in patients at ultra high risk for psychosis. J Affect Disord. 2016;203:101-110. doi:10.1016/j.jad.2016.05.053

74. Michel C, Ruhrmann S, Schimmelmann BG, Klosterkötter J, Schultze-Lutter F. Course of clinical high-risk states for psychosis beyond conversion. Eur Arch Psychiatry Clin Neurosci. 2018;268(1): 39-48. doi:10.1007/s00406-016-0764-8

75. Woods SW, Walsh BC, Addington J, et al. Current status specifiers for patients at clinical high risk for psychosis. Schizophr Res. 2014;158(1-3):69-75. doi:10.1016/j.schres.2014.06.022 